\title{
Carborane as a Tunable Tag for Ru Catalysts: Generating an Anion Appended Recyclable and Robust Catalyst Suitable for Non-covalent Binding Concept
}

\author{
Guiyan Liu, Jianzheng Zhang, Bin Wu, Jianhui Wang* \\ Department of Chemistry, College of Science, Tianjin University, \\ Tianjin 300072, P. R. China.
}

Fax: (+86)-22-2740-3475, e-mail: wjh@tju.edu.cn

\section{Table of Contents}

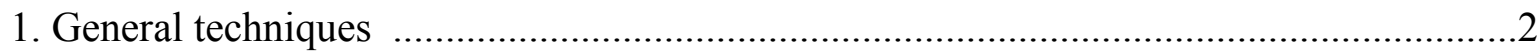

2. Preparation of carborane-tagged ligand 8 ................................................................

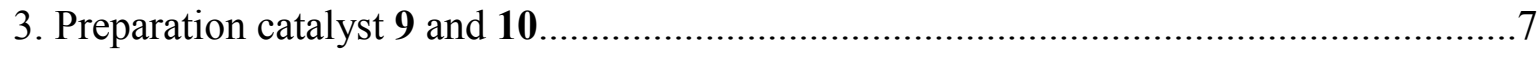

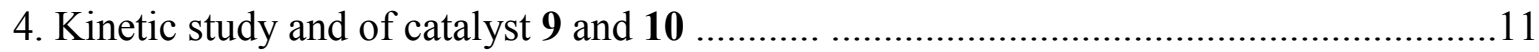

5. Application of $\mathbf{9}$ and $\mathbf{1 0}$ for RCM reactions...............................................................12

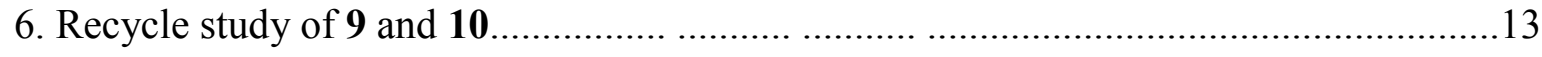

7. Copy of spectroscopy of the prepared compounds......................................................14 


\section{General techniques}

\subsection{Materials}

Unless otherwise noted, all reactions were performed under an atmosphere of dry $\mathrm{N}_{2}$ with oven-dried glassware and anhydrous solvents. THF, benzene, hexanes and diethyl ether were distilled from sodium/benzophenone under a $\mathrm{N}_{2}$ atmosphere. $\mathrm{CH}_{2} \mathrm{Cl}_{2}$ was dried over $\mathrm{CaH}_{2}$, and distilled prior to use. All other solvents were dried over 4-8 $\AA$ mesh molecular sieves (Aldrich) and were either saturated with dry argon or degassed before use. Reactions were monitored by analytical thin layer chromatography on $0.20 \mathrm{~mm}$ Anhui Liangchen silica gel plates and spots were detected by UV-absorption. Silica gel (200-300 mesh) (from Anhui Liangchen Chem. Company, Ltd.) was used for flash chromatography. Dienes $\mathbf{1 1}^{1}$ $13^{2}, 15^{3}, 17^{4}, 19^{5}, 21^{6}, \mathbf{2 3}^{7}, \mathbf{2 5 ^ { 8 }}, \mathbf{2 7 ^ { 9 }}, \mathbf{2 9}^{10}$ were synthesized and purified according to the literature procedures. $\mathrm{BMI}^{\mathrm{P}} \mathrm{F}_{6}$ was prepared and purified as reported previously and dried under high vacuum at $70{ }^{\circ} \mathrm{C}$ overnight to remove trace amount of water ${ }^{11}$. Grubbs catalyst II was prepared according literature method ${ }^{12}$. All other chemicals or reagents were obtained from commercial sources.

\footnotetext{
${ }^{1}$ Van Ornum, S. G. V.; Bruendl, M. M.; Cao, H.; Reddy, M.; Grubisha, D. S.; Bennett, D. W.; Cook, J. M. J. Org. Chem.; 2000, 65, 1957-1971.

${ }^{2}$ Riggi, D. I.; Surzur, J.-M.; Bertrand, M.P. Tetrahedron, 1988, 44,7119-7125.

${ }^{3}$ Moore, J. D.; Byrne, R. J.; Vedantham, P.; Flynn, D. L.; Hanson, P. R. Org. Lett. 2003, 5, 4241-4244.

${ }^{4}$ Edwards, P. G.; Paisey, S.J.; Tooze, R. P.; J. Chem. Soc. Perkin Trans. 2000, 3122-3128.

${ }^{5}$ Garst, M. E.; Bonfiglio, J. N.; Marks, J. J. Org. Chem., 1982, 47,1494-1500.

${ }^{6}$ Otterlo, W. A. L.; Ngidi, E. L.; Koning, C. B. ; Tetrahedron Lett. 2003, 44, 6483-6486.

${ }^{7}$ Yao, Q.; Zhang, Y.; J. Am. Chem. Soc. 2004, 126, 74-75.

${ }^{8}$ Otterlo, W. A. L.; Ngidi, E. L.; Kuzvidza, S.; Morgans, G. L.Morgans, S. S. Moleele; Koning, C. B. ; Tetrahedron, 2005, 61, 9996-10006.

${ }^{9}$ Kim, S.-H.; Zuercher, W. J.; Bowden, N. B.; Grubbs, R. H. J. Org. Chem., 1996, 61,1073-1081.

${ }^{10}$ Barbot, F.; Dauphin, B.; Miginiac, P.; Synthesis, 1985, 768-770.

${ }^{11}$ Huddleston, J. G.; Willauer, H. D.; Swatloski, R. P.; Visser A. E.; Rogers, R. D. Chem. Commun., 1998, 1765-1766.

12 (a)Scholl, M.; Ding, S.; Lee, C. W.; Grubbs, R. H. Org. Lett. 1999, 1, 953-956. (b) Trnka, T. M., Morgan, J. P.; Sanford, M. S.; Willhelm, T. E.; Scholl, M.; Choi, T.-L.; Ding, S.; Day, M. W.; Grubbs, R. H. J. Am. Chem. Soc. 2003, 125, 2546-2558.
} 


\subsection{Spectroscopic Procedures}

${ }^{1} \mathrm{H}-\mathrm{NMR},{ }^{13} \mathrm{C}-\mathrm{NMR},{ }^{11} \mathrm{~B}-\mathrm{NMR}$ spectra were acquired in $\mathrm{CDCl}_{3}$ on Varian 200 and Varian 400 spectrometers. If not otherwise noted, chemical shift values of are reported as values in ppm relative to residual $\mathrm{CHCl}_{3}\left(\delta\right.$ 7.26) for ${ }^{1} \mathrm{H}-\mathrm{NMR}$ spectra, relative to $\mathrm{CDCl}_{3}(77.16 \mathrm{ppm})$ for ${ }^{13} \mathrm{C}-\mathrm{NMR}$ spectra, and relative to $\mathrm{BF}_{3} \mathrm{OEt}_{2}(0.00 \mathrm{ppm})$ for ${ }^{11} \mathrm{~B}-\mathrm{NMR}$ spectra. Multiplicities are described using the following abbreviations: $\mathrm{s}=$ singlet, $\mathrm{d}=$ doublet, $\mathrm{t}=$ triplet, $\mathrm{q}=$ quartet, $\mathrm{m}=$ multiplet, $\mathrm{br}=$ broad. Coupling constants $(J)$ are quoted in Hz. at $400 \mathrm{MHz}$ for ${ }^{1} \mathrm{H}$. Infrared spectra were recorded on a Perkin-Elmer Model 1600 FT-IR spectrophotometer and Nicolet Magna 550 FT-IR spectrophotometer. Elemental analyses were determined in house at Nankai University using a Perkin-Elmer-2400C instrument. Conversions was obtained by HPLC analysis of the sample on Agilent 1100 using Eclipse XDB-C $8(4.6 \times 150 \mathrm{~mm})$ column $\left(\mathrm{CH}_{3} \mathrm{OH} / \mathrm{H}_{2} \mathrm{O}=4: 1\right)$.

\subsection{Synthetic Procedures}

All experiments were carried out in Synthware glass round-bottom flasks of $100 \mathrm{~mL}$ capacity, equipped with magnetic stirring bars and high vacuum Teflon valves. All known compounds among the products were identified by comparing their IR and ${ }^{1} \mathrm{H}$ NMR spectra with those of authentic samples.

\section{Preparation of carborane-tagged ligand 8}

2.1 Synthesis of 2-hydroxy-4-(prop-2-ynyloxy)benzaldehyde ${ }^{13}$

\footnotetext{
${ }^{13}$ Faulques, M.; Rene, L.; Royer, R.; Averbeck, D.; Moradi, M. Eur. J. Med. Chem. Chim. Ther., 1983, 18, 914.
} 


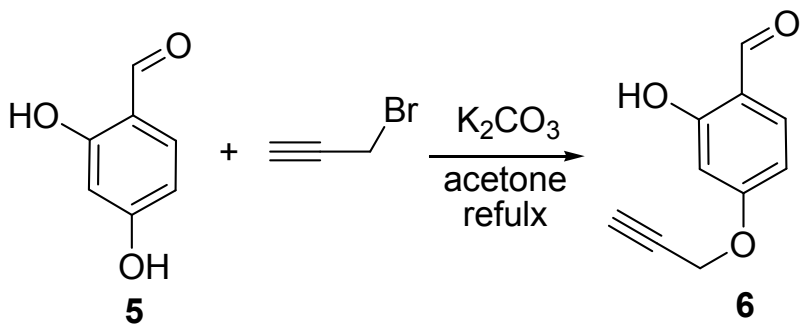

A flask was charged with dry acetone $(30 \mathrm{ml}), 2$,4-dihydroxybenzaldehyde 5 (2.80 g, 20 mmol, 1 equiv) and potassium carbonate $(2.80 \mathrm{~g}, 20 \mathrm{mmol}, 1$ equiv). The mixture was heated to $50{ }^{\circ} \mathrm{C}$, to this reaction mixture, 3-bromoprop-1-yne (2.4 g, $20 \mathrm{mmol}, 1$ equiv) was slowly added via a syringe. Then, the reaction mixtures were stirred for $3 \mathrm{~h}$ under reflux. After cooling to room temperature, the mixtures were concentrated under vacuum and dichloromethane $(100 \mathrm{ml})$ was added. The organic layer was washed four times with water, dried over magnesium sulfate, filtered and concentrated. Purification by silica gel chromatography (pentanes : $\mathrm{CH}_{2} \mathrm{Cl}_{2}, 1: 1$ ) afforded the desired product $\mathbf{6}$ as a white solid (1.60g, $9.00 \mathrm{mmol}, 45 \%)$.

Analytical Data. Calcd (found) for: $\mathrm{C}_{10} \mathrm{H}_{8} \mathrm{O}_{3} \mathrm{C}, 68.18$ (68.54); $\mathrm{H}, 4.58$ (4.55).

${ }^{1} \mathrm{H}-\mathrm{NMR}\left(400 \mathrm{MHz}, \mathrm{CDCl}_{3}\right): \delta(\mathrm{ppm}): 2.58(\mathrm{~s}, 1 \mathrm{H}, \mathrm{CH}), 4.73\left(\mathrm{~s}, 2 \mathrm{H}, \mathrm{CH}_{2}\right), 6.52(\mathrm{~s}, 1 \mathrm{H}$, $\mathrm{CHar}), 6.60\left(\mathrm{~d}, 1 \mathrm{H},{ }^{3} J_{(\mathrm{H}, \mathrm{H})}=8.8 \mathrm{~Hz}, \mathrm{CH} \mathrm{ar}\right), 7.46\left(\mathrm{~d}, 1 \mathrm{H},{ }^{3} J_{(\mathrm{H}, \mathrm{H})}=8.4 \mathrm{~Hz}, \mathrm{CHar}\right), 9.73$ (s,

1H, OH), 11.46(s, 1H, CHO)

${ }^{13} \mathrm{C}-\mathrm{NMR}\left(100 \mathrm{MHz}, \mathrm{CDCl}_{3}\right)$ ): 56.0, 76.5, 76.9, 101.8, 108.6, 115.6, 135.3, 164.2, 164.5, 194.5.

IR (KBr): v 3241, 2846, 2129, 1636, 1508, 1382, 1329, 1287, 1226, 188, 1124, 1012, 976, $835,804,754,702,644,512 \mathrm{~cm}^{-1}$.

\subsection{2-isopropoxy-4-(prop-2-ynyloxy) benzaldehyde}




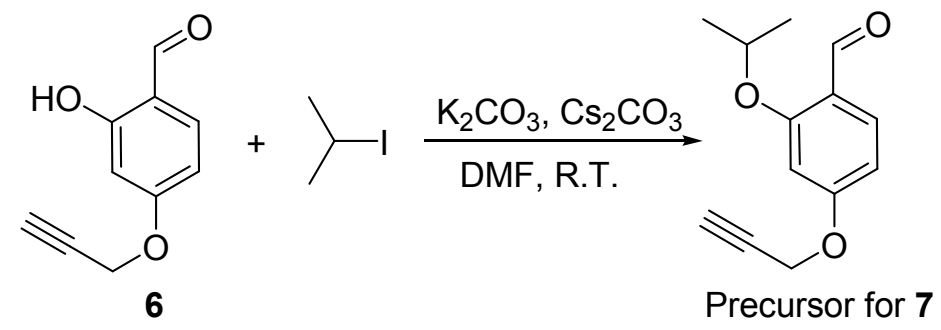

To a solution of 2-hydroxy-4-(prop-2-ynyloxy)benzaldehyde $(0.11 \mathrm{~g}, 0.625 \mathrm{mmol})$ and isopropyl iodine $\left(0.21 \mathrm{~g}, 1.25 \mathrm{mmol}, 2\right.$ equiv) in anhydrous $\mathrm{DMF}(20 \mathrm{~mL}), \mathrm{K}_{2} \mathrm{CO}_{3}(0.173 \mathrm{~g}$, $1.25 \mathrm{mmol})$ and $\mathrm{Cs}_{2} \mathrm{CO}_{3}(0.041 \mathrm{~g}, 0.125 \mathrm{mmol})$ was added. The resulting mixture was stirred at room temperature for $10 \mathrm{~h}$. Then, the mixture was concentrated under vacuum, 30 $\mathrm{mL}$ water was added to the residue, the mixture was then extracted with $\mathrm{CH}_{2} \mathrm{Cl}_{2}(3 \times 15$ ml). The combined organic extracts were washed two times with brine, dried over magnesium sulfate, filtered and concentrated under vacuum. Purification by flash column chromatography on silica (pentanes/ $\mathrm{CH}_{2} \mathrm{Cl}_{2}, 1: 1$ ) gave the desired product as a saffron yellow solid (0.12 g, $0.55 \mathrm{mmol}, 88 \%$ ) (Precursor for 7 )

Analytical Data. Calcd (found) for: $\mathrm{C}_{13} \mathrm{H}_{14} \mathrm{O}_{3} \mathrm{C}, 71.54$ (71.44); H, 6.47(6.55).

${ }^{1} \mathrm{H}-\mathrm{NMR}\left(400 \mathrm{MHz}, \mathrm{CDCl}_{3}\right) \delta(\mathrm{ppm}): 1.37\left(\mathrm{~d},{ }^{3} J(\mathrm{H}, \mathrm{H})=6.0 \mathrm{~Hz}, 6 \mathrm{H}, \mathrm{CH}_{3}\right), 2.57(\mathrm{~s}, 1 \mathrm{H}$, $\mathrm{CH}), 4.60\left(\mathrm{~m},{ }^{3} J(\mathrm{H}, \mathrm{H})=6.0 \mathrm{~Hz}, 1 \mathrm{H}, \mathrm{CH}\right), 4.72(\mathrm{~s}, 2 \mathrm{H}, \mathrm{CH}), 6.52(\mathrm{~s}, 1 \mathrm{H}, \mathrm{CHar}), 6.55(\mathrm{~d}$, $\left.{ }^{3} J(\mathrm{H}, \mathrm{H})=8.8 \mathrm{~Hz}, 1 \mathrm{H}, \mathrm{CHar}\right), 7.77\left(\mathrm{~d},{ }^{3} J(\mathrm{H}, \mathrm{H})=8.8 \mathrm{~Hz}, 1 \mathrm{H}, \mathrm{CHar}\right), 10.30(\mathrm{~s}, 1 \mathrm{H}, \mathrm{CHO})$. ${ }^{13} \mathrm{C}-\mathrm{NMR}\left(100 \mathrm{MHz}, \mathrm{CDCl}_{3}\right) \delta: 21.8,55.8,71.1,76.3,76.7,100.6,106.5,120.3,130.1$, $162.1,163.7,188.6 \mathrm{ppm}$.

$\mathrm{IR}(\mathrm{KBr}): v$ 3210, 2975, 2933, 2857, 2126, 1728, 1668, 1596, 1499, 1440, 1398, 1346, $1295,1262,1189,1122,1032,825,757,709,657,607,551,495 \mathrm{~cm}^{-1}$ 


\subsection{2-isopropoxy-4-(prop-2-ynyloxy)-1-vinylbenzene}

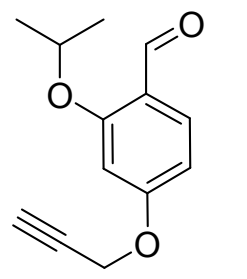

Precursor for 7

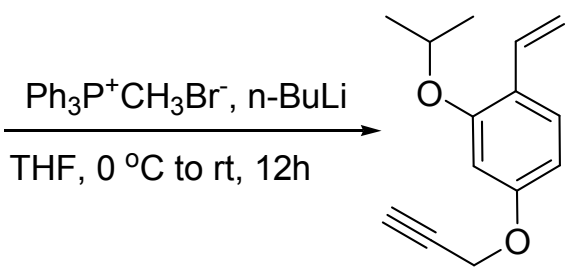

7

To a mixture of $\mathrm{Ph}_{3} \mathrm{P}^{+} \mathrm{MeBr}^{-}(0.62 \mathrm{~g}, 1.73 \mathrm{mmol})$ and $\mathrm{Et}_{2} \mathrm{O}(20 \mathrm{~mL})$ in a Schlenk flask, 1.04 $\mathrm{mL}$-BuLi $(1.66 \mathrm{M}$ in hexanes, $1.73 \mathrm{mmol})$ under nitrogen at $0{ }^{\circ} \mathrm{C}$. After stirring at $0{ }^{\circ} \mathrm{C}$ for $2 \mathrm{~h}$, ethyl ether was removed under vacuum, $20 \mathrm{~mL}$ THF was added to the residue to form a clear solution. To this solution, 2-isopropoxy-4-(prop-2-ynyloxy)benzaldehyde $(0.19 \mathrm{~g}, 0.87 \mathrm{mmol})$ ( precursor for 7 ) in dry ethyl ether was slowly added at $-15^{\circ} \mathrm{C}$, the reaction mixture was stirred for $12 \mathrm{~h}$ before diluted with $\mathrm{Et}_{2} \mathrm{O}(150 \mathrm{~mL})$. The insoluble material was filtered off and the filtrate was concentrated under vacuum. The crude product was purified by flash column chromatography on silica using pentanes $/ \mathrm{CH}_{2} \mathrm{Cl}_{2}(1: 1)$ as eluant to provide 2-isopropoxy-4-(prop-2-ynyloxy)-1-vinylbenzene 7 (0.17 g, $0.79 \mathrm{mmol}$, 91\%) as a yellow oil.

Analytical Data. Calcd (found) for: $\mathrm{C}_{14} \mathrm{H}_{16} \mathrm{O}_{2} \mathrm{C}, 77.75$ (77.64); H, 7.46 (7.35).

${ }^{1} \mathrm{H}-\mathrm{NMR}\left(400 \mathrm{MHz}, \mathrm{CDCl}_{3}\right) \delta(\mathrm{ppm}): 1.36\left(\mathrm{~d},{ }^{3} J(\mathrm{H}, \mathrm{H})=6.0 \mathrm{~Hz}, 6 \mathrm{H}, \mathrm{CH}_{3}\right), 2.55(\mathrm{~s}, 1 \mathrm{H}$, $\mathrm{CH}), 4.52\left(\mathrm{~m},{ }^{3} J(\mathrm{H}, \mathrm{H})=6.0 \mathrm{~Hz}, 1 \mathrm{H}, \mathrm{CH}\right), 4.69\left(\mathrm{~s}, 2 \mathrm{H}, \mathrm{CH}_{2}\right), 5.14\left(\mathrm{~d}, 1 \mathrm{H},{ }^{3} J(\mathrm{H}, \mathrm{H})=12.0 \mathrm{~Hz}\right.$ $\left.=\mathrm{CH}_{2}\right), 5.63\left(\mathrm{~d}, 1 \mathrm{H},{ }^{3} J(\mathrm{H}, \mathrm{H})=16.8 \mathrm{~Hz}=\mathrm{CH}_{2}\right), 6.54(\mathrm{~s}, 1 \mathrm{H}, \mathrm{CHar}) 6.55(\mathrm{~d}, 1 \mathrm{H}, \mathrm{J}(\mathrm{H}, \mathrm{H})=$ $8.8 \mathrm{~Hz}, \mathrm{CHar}), 6.97\left(\mathrm{dd},{ }^{3} J(\mathrm{H}, \mathrm{H})=16.8 \mathrm{~Hz},{ }^{3} J(\mathrm{H}, \mathrm{H})=12.0 \mathrm{~Hz}, 1 \mathrm{H},=\mathrm{CH}\right), 7.42\left(\mathrm{~d},{ }^{3} J(\mathrm{H}, \mathrm{H})\right.$ $=8.8 \mathrm{~Hz}, 1 \mathrm{H}, \mathrm{CHar})$.

${ }^{13} \mathrm{C}-\mathrm{NMR}\left(100 \mathrm{MHz}, \mathrm{CDCl}_{3}\right) \delta: 22.3,56.1,71.1,75.7,76.9,106.4,112.6,122.1,127.2$, $127.4,131.6,156.4,158.49 \mathrm{ppm}$. 
$\operatorname{IR}(\mathrm{KBr}) v:$ 3283, 3083, 2975, 2929, 2873, 2022, 1610, 1577, 1497, 1374, 1290, 1261, $1181,1110,1045,996,905,806,683,631,501,474,443 \mathrm{~cm}^{-1}$

2.4 synthesis of 1-(3-Isopropoxy-4-vinyl-phenoxy)-methyl-1,2-closo-1,2-carborane 8

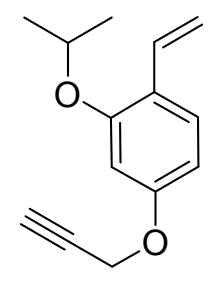

7

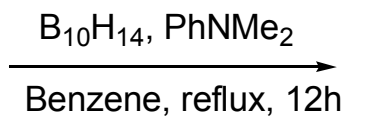

- $=\mathrm{BH}$

$\cdot=\mathrm{CH}$, or $\mathrm{C}$

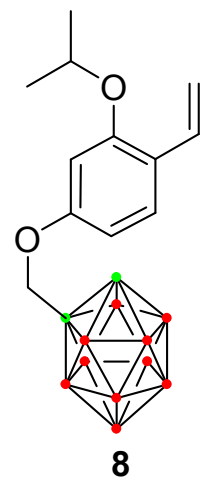

A flask was charged with 2-isopropoxy-4-(prop-2-ynyloxy)-1-vinylbenzene (80 mg, 0.37 mmol), $\mathrm{PhNMe}_{2}(80 \mathrm{mg}, 0.66 \mathrm{mmol}), \mathrm{B}_{10} \mathrm{H}_{14}(53 \mathrm{mg}, 0.43 \mathrm{mmol})$ and dry benzene $(30 \mathrm{~mL})$ under $\mathrm{N}_{2}$. The resulting mixture was refluxed for $8 \mathrm{~h}$. The mixture was concentrated under vacuum. The residue was purified by flash column chromatography on silica using pentanes $/ \mathrm{CH}_{2} \mathrm{Cl}_{2}(2: 1)$ as eluant to give the product 8 as a colourless oil $(0.09 \mathrm{~g}, 0.27$ mmol, $72 \%)$.

Analytical Data. Calcd (found) for: $\mathrm{C}_{14} \mathrm{H}_{26} \mathrm{~B}_{10} \mathrm{O}_{2} \mathrm{C}, 50.27$ (49.89); H, 7.84 (8.17).

${ }^{1} \mathrm{H}-\mathrm{NMR}\left(400 \mathrm{MHz}, \mathrm{CDCl}_{3}\right) \delta(\mathrm{ppm}): 1.36\left(\mathrm{~d},{ }^{3} J(\mathrm{H}, \mathrm{H})=6.0 \mathrm{~Hz}, 6 \mathrm{H}, \mathrm{CH}_{3}\right), 2.09-2.60$ (broad, 1H, CH, 10H, BH), 4.06(s, $1 \mathrm{H}, \mathrm{CH}), 4.39\left(\mathrm{~d},{ }^{3} J(\mathrm{H}, \mathrm{H})=10.0 \mathrm{~Hz}, 2 \mathrm{H}, \mathrm{CH}_{2}\right), 4.53$ $\left(\mathrm{m}, 1 \mathrm{H},{ }^{3} J(\mathrm{H}, \mathrm{H})=6.0 \mathrm{~Hz}, \mathrm{CH}\right), 5.17\left(\mathrm{~d}, 1 \mathrm{H},{ }^{3} J(\mathrm{H}, \mathrm{H})=11.2,=\mathrm{CH}_{2}\right), 5.63\left(\mathrm{~d}, 1 \mathrm{H},{ }^{3} J(\mathrm{H}, \mathrm{H})=\right.$ $\left.18.0 \mathrm{~Hz},=\mathrm{CH}_{2}\right), 6.39(\mathrm{~s}, 1 \mathrm{H}, \mathrm{CHar}), 6.40\left(\mathrm{~d}, 1 \mathrm{H},{ }^{3} J(\mathrm{H}, \mathrm{H})=5.6 \mathrm{~Hz}, \mathrm{CHar}\right), 6.97(\mathrm{dd}, 1 \mathrm{H}$, $\left.{ }^{3} J(\mathrm{H}, \mathrm{H})=11.2,{ }^{3} J(\mathrm{H}, \mathrm{H})=17.6 \mathrm{~Hz},=\mathrm{CH}\right), 7.39\left(\mathrm{~d},{ }^{3} J(\mathrm{H}, \mathrm{H})=8.4 \mathrm{~Hz}, 1 \mathrm{H}, \mathrm{CHar}\right)$.

${ }^{13} \mathrm{C}-\mathrm{NMR}\left(100 \mathrm{MHz}, \mathrm{CDCl}_{3}\right) \delta(\mathrm{ppm}): 17.8,53.5,65.1,66.8,66.9,97.6,101.5,108.7,118.8$, $123.2,126.9,152.1,153.4$.

${ }^{11} \mathrm{~B}-\mathrm{NMR}\left(64 \mathrm{MHz}, \mathrm{CDCl}_{3}\right) \delta(\mathrm{ppm}):-3.52,-8.99,-12.23$; 
$\mathrm{IR}(\mathrm{KBr}):$ v 3084, 2978, 2926, 2588, 1605, 1500, 1465, 1425, 1385, 1261, 1186, 1117, 999, $915,805,722,690 \mathrm{~cm}^{-1}$.

\section{Preparation catalyst 9 and 10}

\subsection{Preparation of catalyst 9}

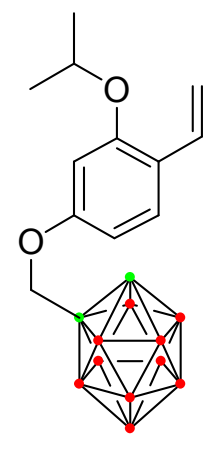

8

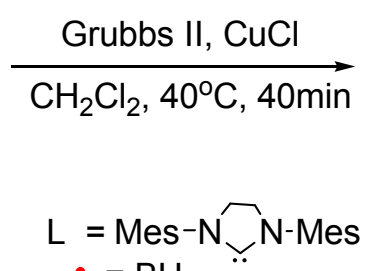

$\cdot=\mathrm{BH}$

$\cdot=\mathrm{CH}$, or $\mathrm{C}$

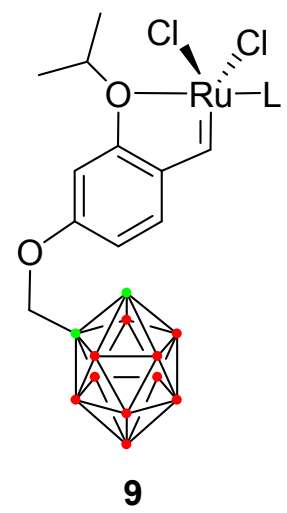

Grubbs catalyst II (424 mg, $0.50 \mathrm{mmol})$ and $\mathrm{CuCl}(50 \mathrm{mg}, 0.50 \mathrm{mmol})$ was added into a flask under $\mathrm{N}_{2}$. A solution of 1-(3-Isopropoxy-4-vinyl-phenoxy)-methyl-1,2-closo-1,2carborane $8(200 \mathrm{mg}, 0.6 \mathrm{mmol})$ in dry dichloromethane $(25 \mathrm{~mL})$ was poured into the reaction mixture under $\mathrm{N}_{2}$. The resulting mixtures were then refluxed for $20 \mathrm{~min}$. After cooling down to room temperature, the reaction mixtures were filtered to collect the filtrate. Solvent from the filtrate was evaporated off under vacuum to give a residue. The residue was purified by flash column chromatography on silica using $\mathrm{CH}_{2} \mathrm{Cl}_{2}$ as elutant to give the desired product 9 as a green crystalline solid (311 $\mathrm{mg}, 0.39 \mathrm{mmol}, 78 \%$ ).

Analytical Data. Calcd (found) for: $\mathrm{C}_{34} \mathrm{H}_{50} \mathrm{~B}_{10} \mathrm{Cl}_{2} \mathrm{~N}_{2} \mathrm{O}_{2} \mathrm{Ru} \mathrm{C}, 51.12$ (51.21); H, 6.31 (6.32); $\mathrm{N}, 3.51(3.71)$.

${ }^{1} \mathrm{H}-\mathrm{NMR}\left(400 \mathrm{MHz}, \mathrm{CDCl}_{3}\right) \delta(\mathrm{ppm}): 1.26\left(\mathrm{~d},{ }^{3} J(\mathrm{H}, \mathrm{H})=6.0 \mathrm{~Hz}, 6 \mathrm{H}, \mathrm{CH}\right), 0.86-2.81(\mathrm{br}$, 10H, BH), 2.39 (s, 6H, CH $H_{3}, 2.46\left(\mathrm{~s}, 12 \mathrm{H}, \mathrm{CH}_{3}\right), 3.96(\mathrm{~s}, 1 \mathrm{H}$, Cage $(\mathrm{CH})), 4.17\left(\mathrm{~s}, 4 \mathrm{H}, \mathrm{CH}_{2}\right)$, $4.335(\mathrm{~s}, 2 \mathrm{H}, \mathrm{CH}), 4.79\left(\mathrm{~m},{ }^{3} J(\mathrm{H}, \mathrm{H})=6.0 \mathrm{~Hz}, \mathrm{H}, \mathrm{CH}\right), 6.28(\mathrm{~s}, 1 \mathrm{H}, \mathrm{CHar}), 6.31(\mathrm{~d}, \mathrm{~J}(\mathrm{H}, \mathrm{H})=$ 
$8.8 \mathrm{~Hz}, \mathrm{H}, \mathrm{CHar}), 6.83\left(\mathrm{~d},{ }^{3} J(\mathrm{H}, \mathrm{H})=8.4 \mathrm{~Hz}, 1 \mathrm{H}, \mathrm{CHar}\right), 7.06$ (s, 4H, CHar), 16.09(s,1H,Ru$H)$.

${ }^{13} \mathrm{C}-\mathrm{NMR}\left(100 \mathrm{MHz}, \mathrm{CDCl}_{3}\right) \delta(\mathrm{ppm}): 16.8,21.7,23.0,47.2,53.6,65.1,66.8,71.41,97.2$, $102.5,119.3,125.1,134.5,137.8,149.7,153.9,206.7,282.9$.

${ }^{11} \mathrm{~B}-\mathrm{NMR}\left(64 \mathrm{MHz}, \mathrm{CDCl}_{3}\right) \delta(\mathrm{ppm}):-2.62,-9.04,-11.84$;

$\mathrm{IR}(\mathrm{KBr}) v: 2929,2850,2589,1597,1490,1396,1324,1263,1187,1038,1003$

, 1003, 912, 850, 726, 513, $417 \mathrm{~cm}^{-1}$

\subsection{Preparation of catalyst 10}

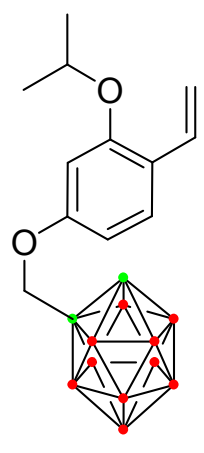

8
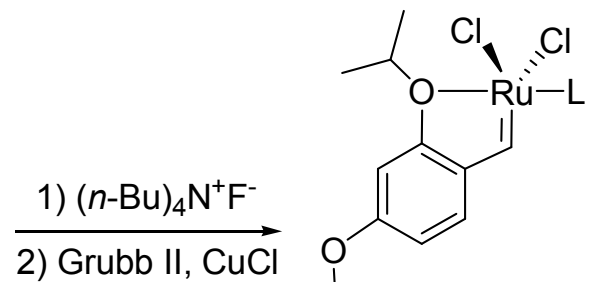

$\mathrm{L}=$ Mes- $-\mathrm{N}_{V} \mathrm{~N}-\mathrm{Mes}$

- = $\mathrm{BH}$

$\cdot=\mathrm{CH}$, or $\mathrm{C}$

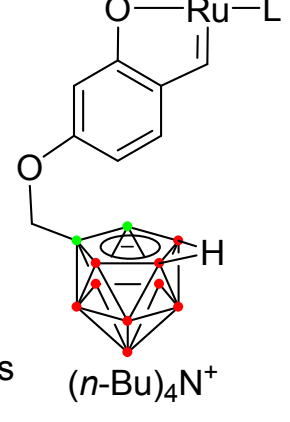

10

A solution of 1-(3-Isopropoxy-4-vinyl-phenoxy)-methyl-1,2-closo-1,2-carborane (8) (0.11 g, $0.33 \mathrm{mmol})$ in THF $(15 \mathrm{~mL})$ was treated with $(\mathrm{n}-\mathrm{Bu})_{4} \mathrm{~N}^{+} \mathrm{F}^{-}(0.49 \mathrm{~g}, 1.88 \mathrm{mmol})$, and the resulting mixture was stirred for $3 \mathrm{~h}$ at room temperature. Water $(15 \mathrm{~mL})$ was added and the organic layer separated. The organic layer was washed further with water $(2 \times 15 \mathrm{~mL})$, dried over dry magnesium sulfate, filtered and concentrated. Purification by silica gel chromatography (pentanes : $\mathrm{CH}_{2} \mathrm{Cl}_{2}, 1: 2$ ) gave the desired cage-opening product (precursor for 10 ) as a yellowy solid (0.18 g, $0.32 \mathrm{mmol}, 97 \%)$

Analytical Data. Calcd (found) for: $\mathrm{C}_{30} \mathrm{H}_{61} \mathrm{~B}_{9} \mathrm{NO}_{2} \mathrm{C}, 63.76$ (64.03); H, 10.88 (11.01); N, $2.48(2.35)$ 
${ }^{1} \mathrm{H}-\mathrm{NMR}\left(400 \mathrm{MHz}, \mathrm{CDCl}_{3}\right) \delta(\mathrm{ppm}):-2.53(\mathrm{~s}, 1 \mathrm{H}, \mathrm{BH}), 0.97\left(\mathrm{~m},{ }^{3} J(\mathrm{H}, \mathrm{H})=7.8 \mathrm{~Hz}, 12 \mathrm{H}\right.$, $\left.\mathrm{CH}_{3}\right), 1.31\left(\mathrm{~d},{ }^{3} J(\mathrm{H}, \mathrm{H})=5.6 \mathrm{~Hz}, 6 \mathrm{H}, \mathrm{CH}_{3}\right), 1.41\left(\mathrm{~m},{ }^{3} J(\mathrm{H}, \mathrm{H})=8.0 \mathrm{~Hz}, 8 \mathrm{H}, \mathrm{CH}_{2}\right), 1.61(\mathrm{~m}$, $\left.{ }^{3} J(\mathrm{H}, \mathrm{H})=5.6 \mathrm{~Hz}, 8 \mathrm{H}, \mathrm{CH}\right), 2.06(\mathrm{~s}, 1 \mathrm{H}, \mathrm{CH}), 3.11\left(\mathrm{~m},{ }^{3} \mathrm{~J}(\mathrm{H}, \mathrm{H})=8.0 \mathrm{~Hz}, 8 \mathrm{H}, \mathrm{CH}_{2}\right), 3.80$ $\left(\mathrm{d}, 1 \mathrm{H}, \mathrm{O}-\mathrm{CH}_{2}\right), 4.17\left(\mathrm{~d}, 1 \mathrm{H}, \mathrm{O}-\mathrm{CH}_{2}\right), 4.50\left(\mathrm{~m},{ }^{3} J(\mathrm{H}, \mathrm{H})=6.0 \mathrm{~Hz}, 1 \mathrm{H}, \mathrm{CH}\right), 5.05\left(\mathrm{~d},{ }^{3} J(\mathrm{H}, \mathrm{H})=\right.$ $\left.12.0 \mathrm{~Hz}, 1 \mathrm{H},=\mathrm{C} H_{2}\right), 5.59\left(\mathrm{~d},{ }^{3} J(\mathrm{H}, \mathrm{H})=17.6 \mathrm{~Hz}, 1 \mathrm{H},=\mathrm{CH} H_{2}, 6.40\left(\mathrm{~d},{ }^{3} J(\mathrm{H}, \mathrm{H})=7.6 \mathrm{~Hz}, 1 \mathrm{H}\right.\right.$, CHar), $6.43(\mathrm{~s}, 1 \mathrm{H}, \mathrm{CHar}), 6.93\left(\mathrm{dd},{ }^{3} J(\mathrm{H}, \mathrm{H})=17.6 \mathrm{~Hz},{ }^{3} J(\mathrm{H}, \mathrm{H})=11.6 \mathrm{~Hz}, 1 \mathrm{H},=\mathrm{CH}\right), 7.31$ $\left(\mathrm{d},{ }^{3} J(\mathrm{H}, \mathrm{H})=7.6 \mathrm{~Hz}, 1 \mathrm{H}, \mathrm{CHar}\right)$.

${ }^{13} \mathrm{C}-\mathrm{NMR}\left(100 \mathrm{MHz}, \mathrm{CDCl}_{3}\right) \delta(\mathrm{ppm}): 13.6,19.6,22.2,23.9,58.9,71.0,75.6,102.0$, $107.0,111.2,120.5,126.9,131.7,156.1,160.4$.

${ }^{11} \mathrm{~B}-\mathrm{NMR}\left(64 \mathrm{MHz}, \mathrm{CDCl}_{3}\right) \delta(\mathrm{ppm}):-10.5,-17.9,-22.3,-33.1,-37.3$;

$\operatorname{IR}(\mathrm{KBr}) \vee 3083,2965,2933,2874,2526,1602,1498,1463,1378,1295,1264,1185,1112$ $1018,906,841,737 \mathrm{~cm}^{-1}$.

The cage-opening product (339 $\mathrm{mg}, 0.6 \mathrm{mmol}$ ) prepared last step was dissolve in dry $\mathrm{CH}_{2} \mathrm{Cl}_{2}(25 \mathrm{ml})$ to give a clear solution. To this solution, Grubbs catalyst II (424 mg, 0.50 $\mathrm{mmol})$ and $\mathrm{CuCl}(50 \mathrm{mg}, 0.50 \mathrm{mmol})$ was added at room temperature. After stirred and refluxed for $20 \mathrm{~min}$, solvent from the reaction mixture was evaporated under vacuum to give a residue. The residue was purified by flash column chromatography using $\mathrm{CH}_{2} \mathrm{Cl}_{2}$ as elutant to afford the desired product $\mathbf{1 0}$ as a yellow green crystalline solid (329 $\mathrm{mg}, 0.32$ mmol, 64\%).

Analytical Data. Calcd (found) for: $\mathrm{C}_{50} \mathrm{H}_{85} \mathrm{~B}_{9} \mathrm{Cl}_{2} \mathrm{~N}_{3} \mathrm{O}_{2} \mathrm{Ru}$ C, 58.335 (58.68); H, 8.32 (8.59); N, $4.08(3.89)$.

${ }^{1} \mathrm{H}-\mathrm{NMR}\left(400 \mathrm{MHz}, \mathrm{CDCl}_{3}\right) \delta(\mathrm{ppm}):-2.51(\mathrm{~s}, 1 \mathrm{H}, \mathrm{BH}), 0.93\left(\mathrm{~m},{ }^{3} J(\mathrm{H}, \mathrm{H})=6.8 \mathrm{~Hz}, 12 \mathrm{H}\right.$, $\left.\mathrm{CH}_{3}\right), 1.24\left(\mathrm{~d},{ }^{3} J(\mathrm{H}, \mathrm{H})=5.6 \mathrm{~Hz}, 6 \mathrm{H}, \mathrm{CH}_{3}\right), 1.31\left(\mathrm{~m},{ }^{3} J(\mathrm{H}, \mathrm{H})=6.8 \mathrm{~Hz}, 8 \mathrm{H}, \mathrm{CH}_{2}\right), 1.56(8 \mathrm{H}$, 
$\left.\mathrm{CH}_{2}\right), 1.98(\mathrm{~s}, 1 \mathrm{H}, \mathrm{CH}), 2.28\left(\mathrm{~s}, 6 \mathrm{H}, \mathrm{CH}_{3}\right), 2.50\left(\mathrm{~s}, 12 \mathrm{H}, \mathrm{CH}_{3}\right), 3.04\left(\mathrm{t},{ }^{3} J(\mathrm{H}, \mathrm{H})=8.0 \mathrm{~Hz}\right.$, $\left.8 \mathrm{H}, \mathrm{CH}_{2}\right), 3.77(\mathrm{~d}, \mathrm{~J}(\mathrm{H}, \mathrm{H})=10.4 \mathrm{~Hz} 2 \mathrm{H}, \mathrm{CH}), 4.14(\mathrm{~s}, 4 \mathrm{H}, \mathrm{CH}), 4.79\left(\mathrm{~m},{ }^{3} J(\mathrm{H}, \mathrm{H})=6.0\right.$ $\mathrm{Hz}, 1 \mathrm{H}, \mathrm{CH}), 6.31\left(\mathrm{~d},{ }^{3} J(\mathrm{H}, \mathrm{H})=8.8 \mathrm{~Hz}, 2 \mathrm{H}, \mathrm{CHar}\right), 6.71\left(\mathrm{~d},{ }^{3} J(\mathrm{H}, \mathrm{H})=8.4 \mathrm{~Hz}, 1 \mathrm{H}, \mathrm{CHar}\right)$, 7.03 (s, 4H, CHar), 16.13(s, 1H, Ru-.).

${ }^{13} \mathrm{C}-\mathrm{NMR}\left(100 \mathrm{MHz}, \mathrm{CDCl}_{3}\right) \delta(\mathrm{ppm}): 13.8,19.9,21.3,24.2,50.9,59.4,75.2,76.0,101.4$, $107.4,123.8,129.5,130.4,135.1,133.8,141.0,154.5,162.0,213.5,295.9$.

${ }^{11} \mathrm{~B}-\mathrm{NMR}\left(64 \mathrm{MHz}, \mathrm{CDCl}_{3}\right) \delta(\mathrm{ppm}):-11.1,-18.2,-22.7,-33.3,-37.6$;

$\mathrm{IR}(\mathrm{KBr}) v: 2926,2875,2530,1632,1596,1487,1451,1382,1261,1156,1102$ , 1027, 850, 743, 668, 571, $487 \mathrm{~cm}^{-1}$

\section{Kinetic study of 4, 9 and 10 for $R C M$ reaction}

Under $\mathrm{N}_{2}$ gas, a glass vial with a screw-cap septum top was charged with catalyst $[2.50 \mathrm{mg}$ (0.004 mmol) for 4; $3.2 \mathrm{mg}(0.004 \mathrm{mmol})$ for $9 ; 4.1 \mathrm{mg}(0.004 \mathrm{mmol})$ for 10$]$ and $\mathrm{CH}_{2} \mathrm{Cl}_{2}$ $(4.00 \mathrm{~mL})$ to give a $1 \mu \mathrm{mol} / \mathrm{L}$ solution. The solution was then cooled to $0{ }^{\circ} \mathrm{C}$ by ice-water bath (or heated to $30{ }^{\circ} \mathrm{C}$ by water bath for kinetic study at different temperatures). $0.48 \mathrm{~g}$ (2.0 mmol) Diethyldiallyl malonate was then injected into the solution via syringe. Aliquots were taken from the reaction mixture at appropriate times using a syringe and were quenched immediately by $0.1 \mathrm{~mol} / \mathrm{L}$ PEI in $\mathrm{CH}_{2} \mathrm{Cl}_{2}$, and then the resulted solution was passed a short column chromatography to remove the $\mathrm{Ru}$ metal residue using $\mathrm{CH}_{2} \mathrm{Cl}_{2}$ as eluant. Solvent from the collected solution was evaporated under vacuum. The colorless residue was diluted by methanol and water (80:20) for HPLC detection. Conversions were obtained by comparing the ratios of the integrals of the peak area of starting material with those of product. The graph was shown in Figure 1. 
Figure 1 Relative conversion rates of 9 and 10 using $1 \mathrm{~mol} \%$ catalyst in $\mathrm{CH}_{2} \mathrm{Cl}_{2}$ :. a, at $0^{\circ} \mathrm{C}$; b, at $30^{\circ} \mathrm{C}$.
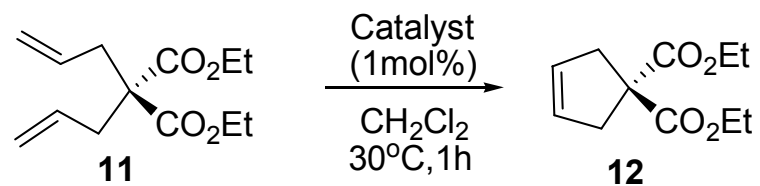

4: $98 \%$

9: $97 \%$

10: $92 \%$

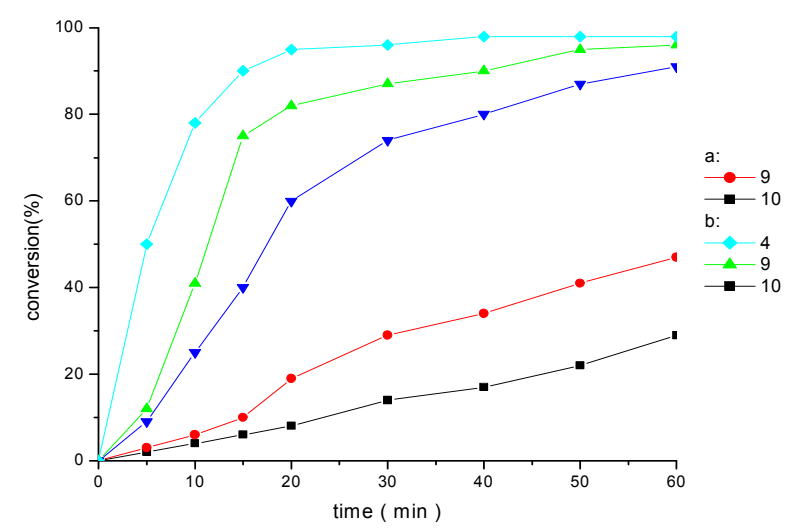

\section{Application of 9 and 10 for $R C M$ reactions}

General procedure for metathesis reactions with $\mathbf{9}$ and $\mathbf{1 0}$ was described as follow:

Catalyst $(0.5 \mathrm{~mol} \%)$ and a solution of the substrate $(0.05 \mathrm{mmol})$ in dry $\mathrm{CH}_{2} \mathrm{Cl}_{2}(2.0 \mathrm{~mL})$ was mixed in a reaction flask under argon. The reaction mixture was stirred at room temperature for 2-12 h. At the end of the reaction (monitored by TLC or GC), were quenched immediately by $0.25 \mathrm{~g} / \mathrm{mL}$ PEI in $\mathrm{CH}_{2} \mathrm{Cl}_{2}$, and then the resulted solution was passed a short column chromatography to remove the $\mathrm{Ru}$ metal residue using $\mathrm{CH}_{2} \mathrm{Cl}_{2}$ as eluant. The crude products was further purified by flash column chromatography (mixture of petroleum ether $/ \mathrm{CH}_{2} \mathrm{Cl}_{2}$ as eluant ) to obtain analytically pure samples of the corresponding products. The results were listed in Table 1 
Table 1. Application of Catalyst 9 and 10 on Different Substrates

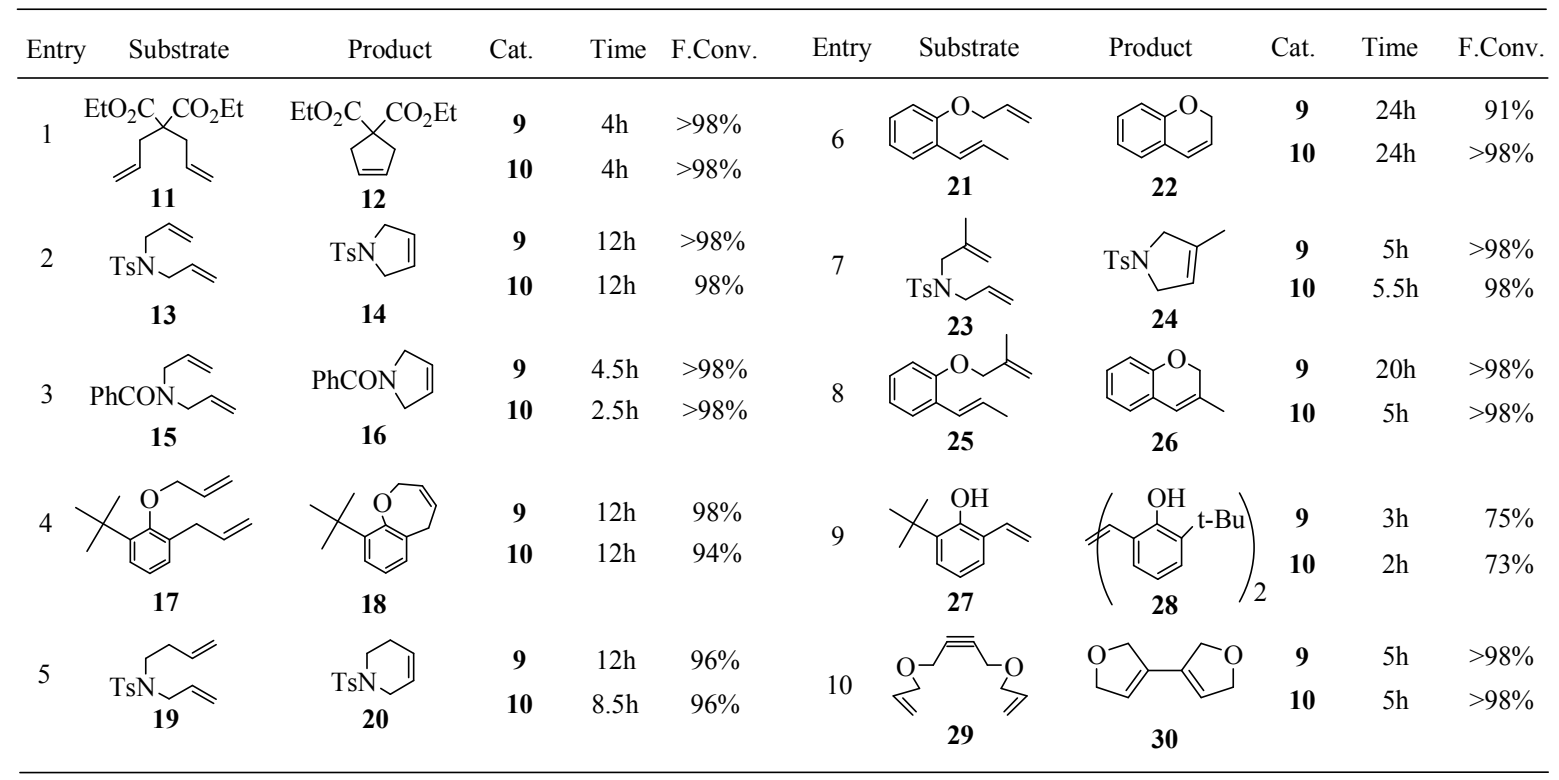

\section{Recycle study of 9 and 10 in ILs.}

General procedure for $\mathrm{RCM}$ reactions in $\mathrm{BMI} \cdot \mathrm{PF}_{6}$ were described as following:

A mini amount $(0.04 \mathrm{~mL}) \mathrm{BMI} \mathrm{PF}_{6}$ was introduced into a Schlenk flask and dried $2 \mathrm{~h}$ under vacuum at $70{ }^{\circ} \mathrm{C}$. After cooled to room temperature, a solution of the catalyst $(3.2 \mathrm{mg}$, $0.004 \mathrm{mmol}$ for $9 ; 4.1 \mathrm{mg}, 0.004 \mathrm{mmol}$ for 10) in $\mathrm{CH}_{2} \mathrm{Cl}_{2}(0.50 \mathrm{~mL})$ was added and the reaction mixture was stirred for $10 \mathrm{~min}$ to give a clear homogenous solution. A solution of $\mathrm{N}, \mathrm{N}$-dially-4-methylbenzenesulfonamide (23) $\left(40 \mathrm{mg}, 0.16 \mathrm{mmol}\right.$ in $\mathrm{CH}_{2} \mathrm{Cl}_{2}(0.50 \mathrm{~mL})$ was then introduced into the reaction mixture and heated to $30{ }^{\circ} \mathrm{C}$ for a period of time. At the end of the reaction, most of solvent from the mixture was evaporated under vacuum (Do not completely remove the $\mathrm{CH}_{2} \mathrm{Cl}_{2}$. Otherwise, the product could precipitate from the reaction mixture, which cause serious separation problem) and the cyclized product was obtained by extraction with hexanes $(2 \times 5 \mathrm{~mL})$. The solvent was evaporated to afford the crude product. After a simple filtration onto a plug of silica gel using ethyl acetate as eluant 
to removal the trace amount of $\mathrm{Ru}$ residue, the sample was subjected for HPLC detection. Conversions were obtained by comparing the ratios of the integrals of starting materials with those of products. The recovered catalyst in ILs was reused for another cycle. Results were shown in Table 1.

Table 1. Recycle Study of Catalyst $\mathbf{9}$ and $\mathbf{1 0}$

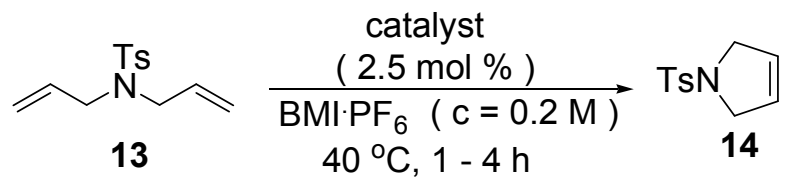

\begin{tabular}{cccccccccccc}
\hline \multirow{2}{*}{ catalyst } & \multicolumn{10}{c}{ cycle ( \% convn. ) } \\
\hline & 1 & 2 & 3 & 4 & 5 & 6 & 7 & 8 & 9 & 10 \\
\hline 9 & $>98 \%$ & $30 \%$ & - & - & - & - & - & - & - & - \\
10 & $>98 \%^{\mathrm{a}}$ & $>98 \%^{\mathrm{a}}$ & $>98 \%^{\mathrm{a}}$ & $>98 \%^{\mathrm{a}}$ & $>98 \%^{\mathrm{a}}$ & $>97 \%^{\mathrm{a}}$ & $>97 \%^{\mathrm{a}}$ & $97 \%^{\mathrm{a}}$ & $97 \%^{\mathrm{b}}$ & $95 \%{ }^{\mathrm{c}}$
\end{tabular}

Reaction time for each run: a, 1h; b, 2h; c, $4 h$.

\section{Copy of spectroscopy}




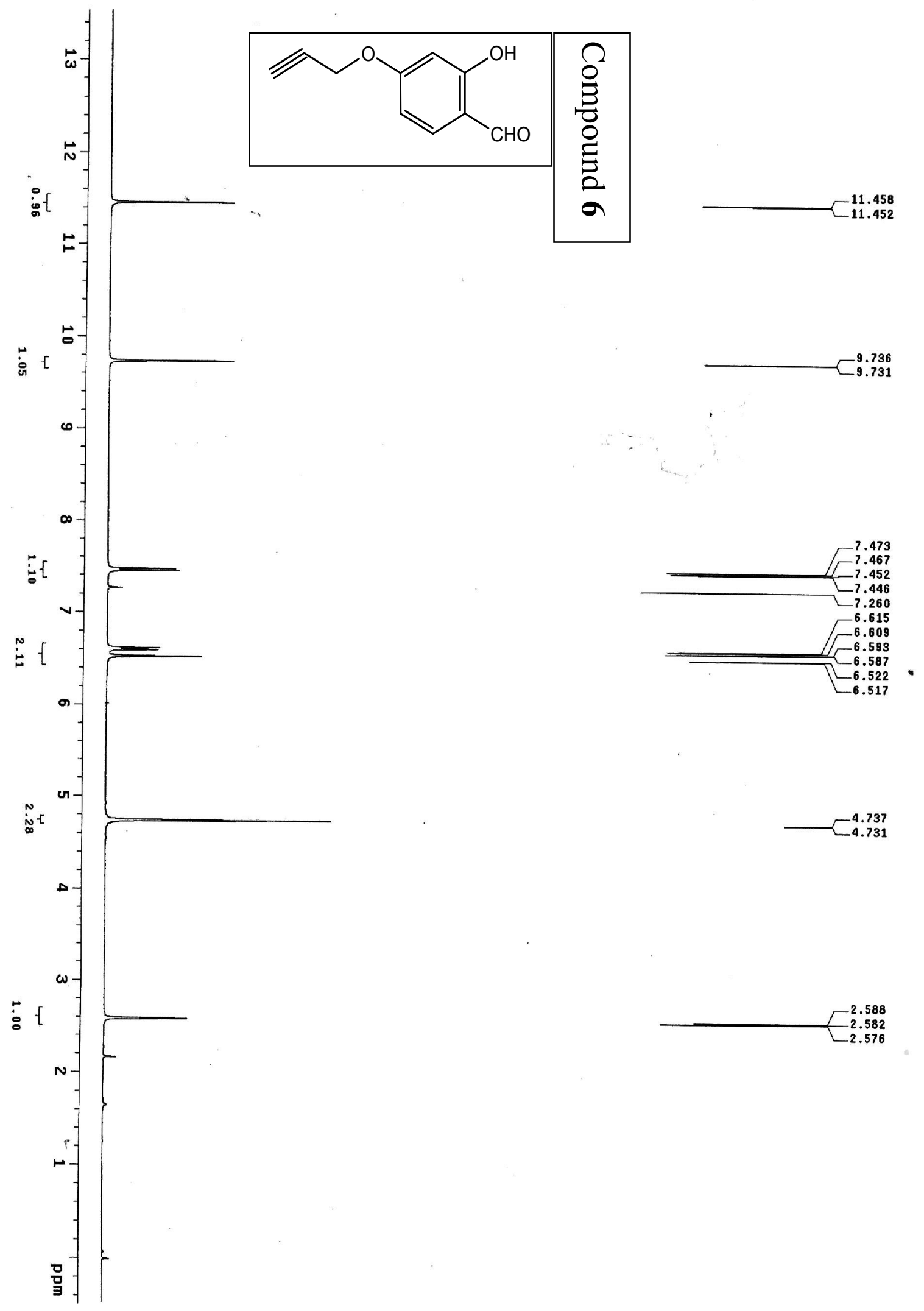




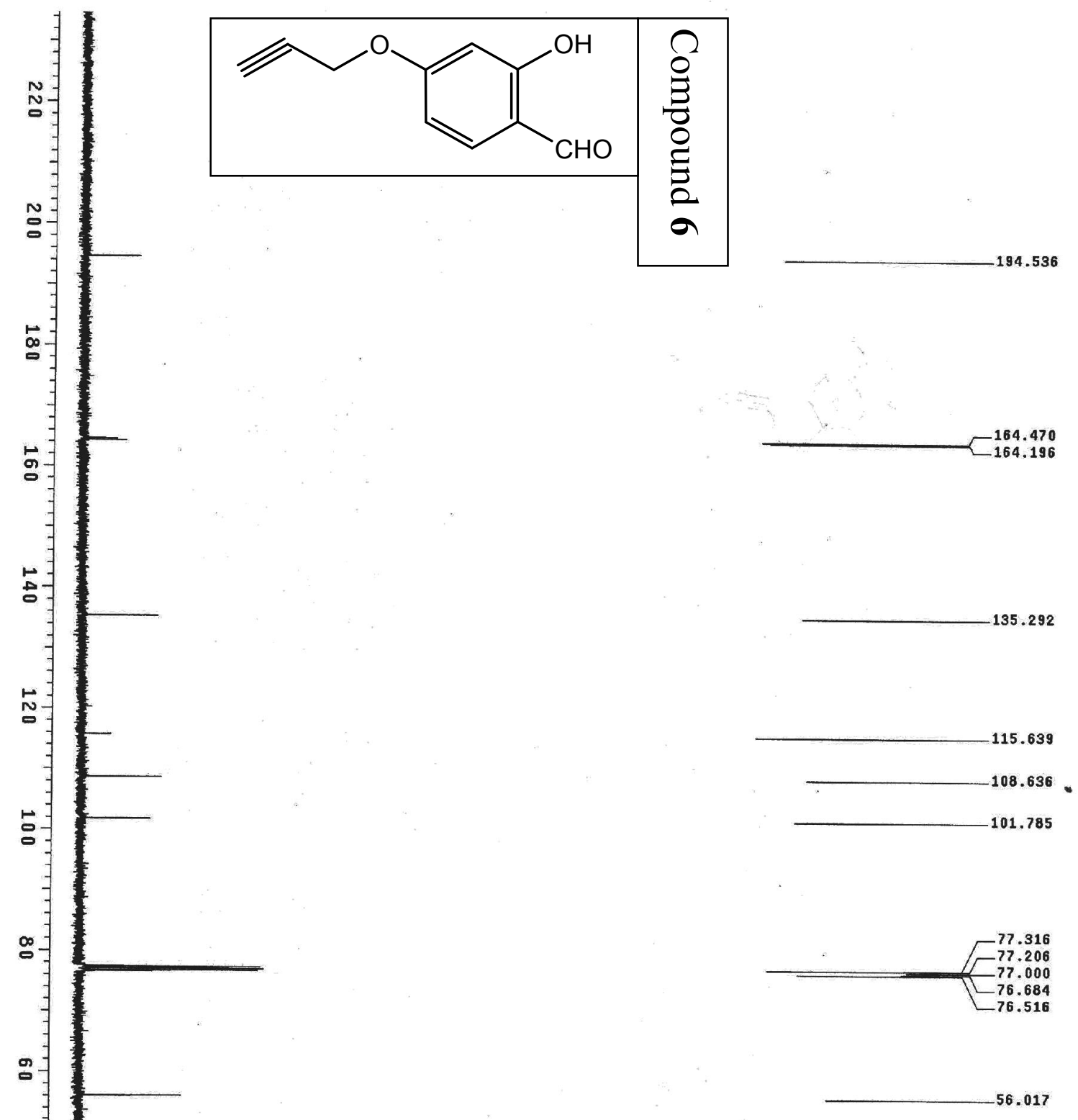




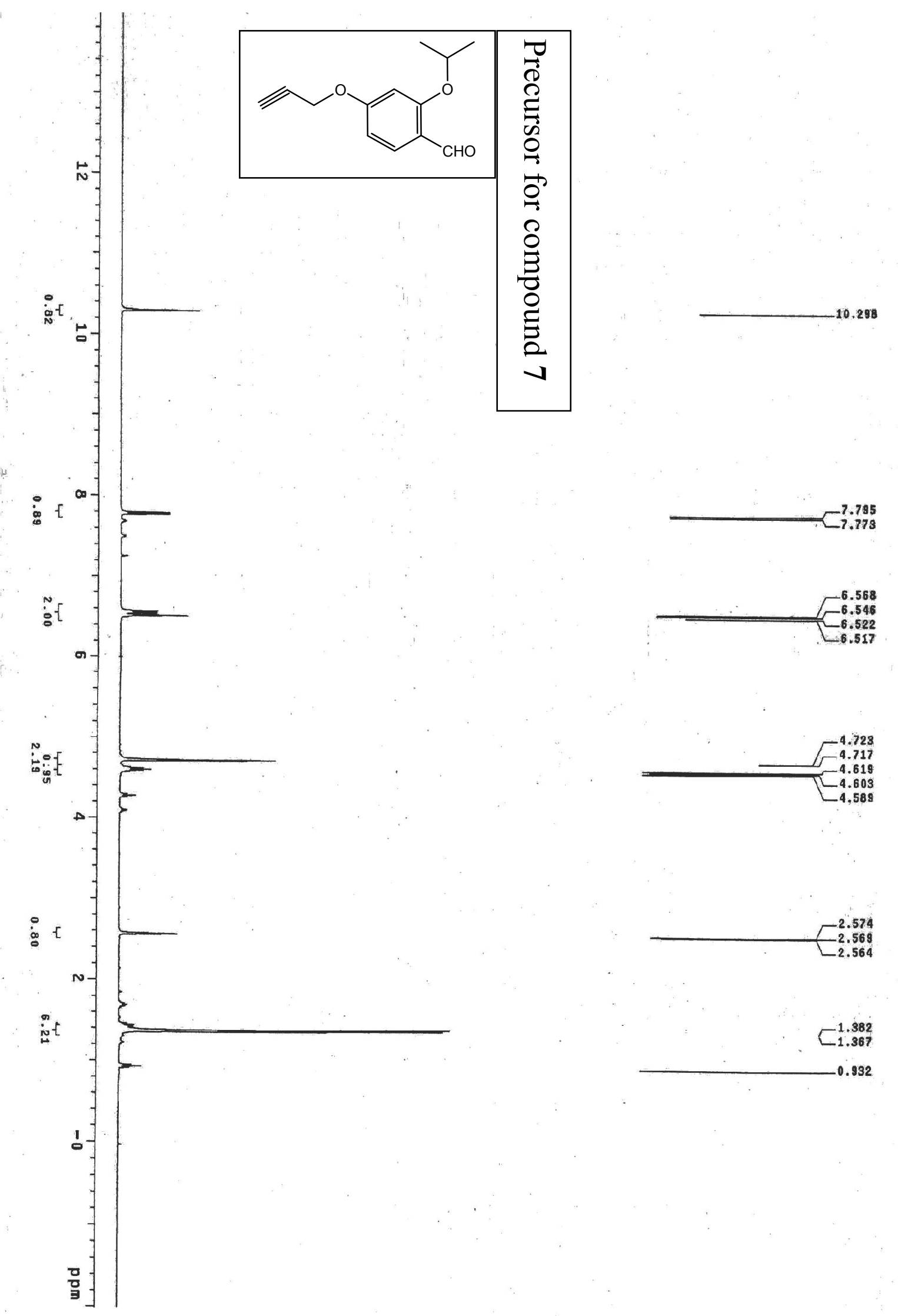




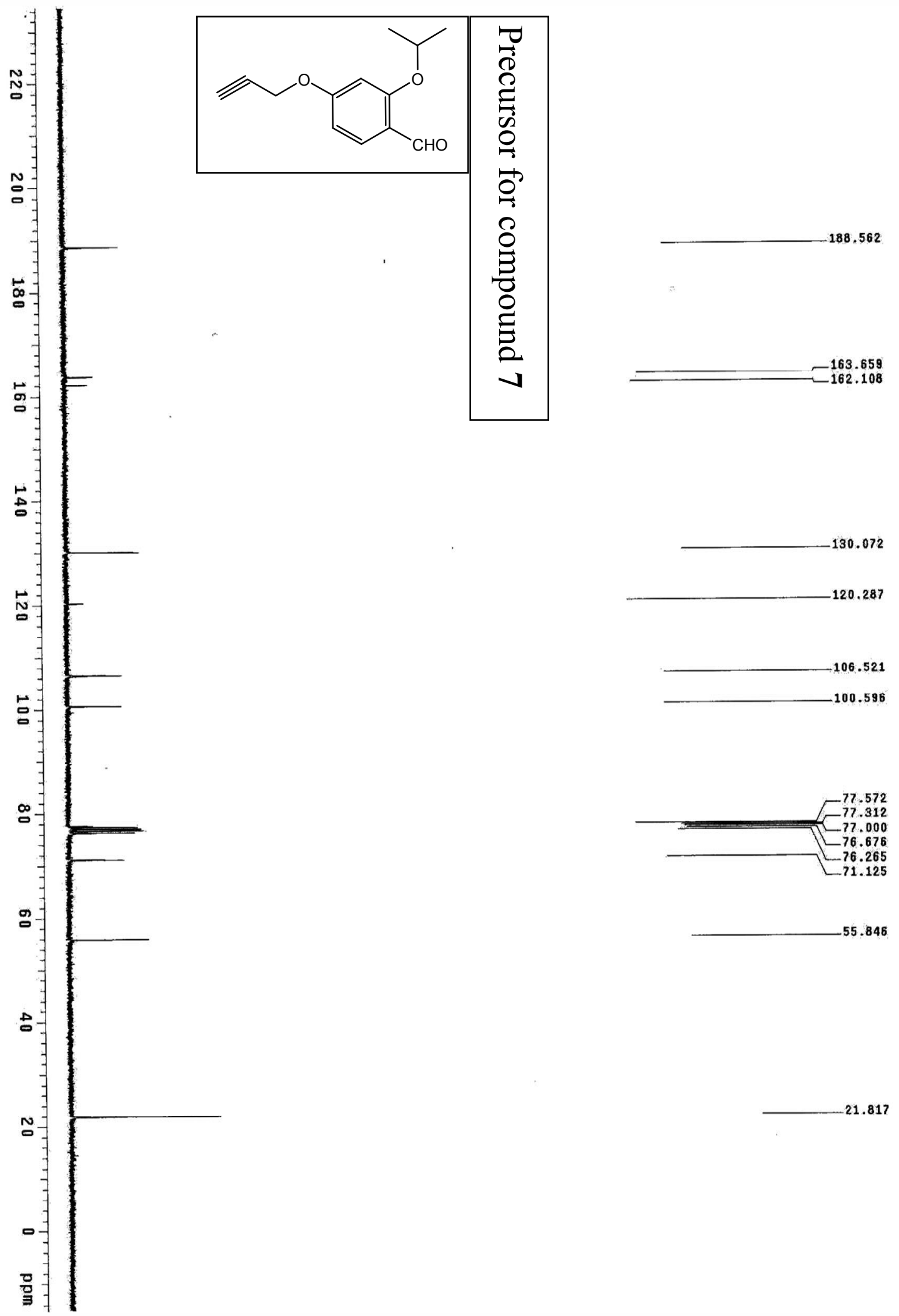




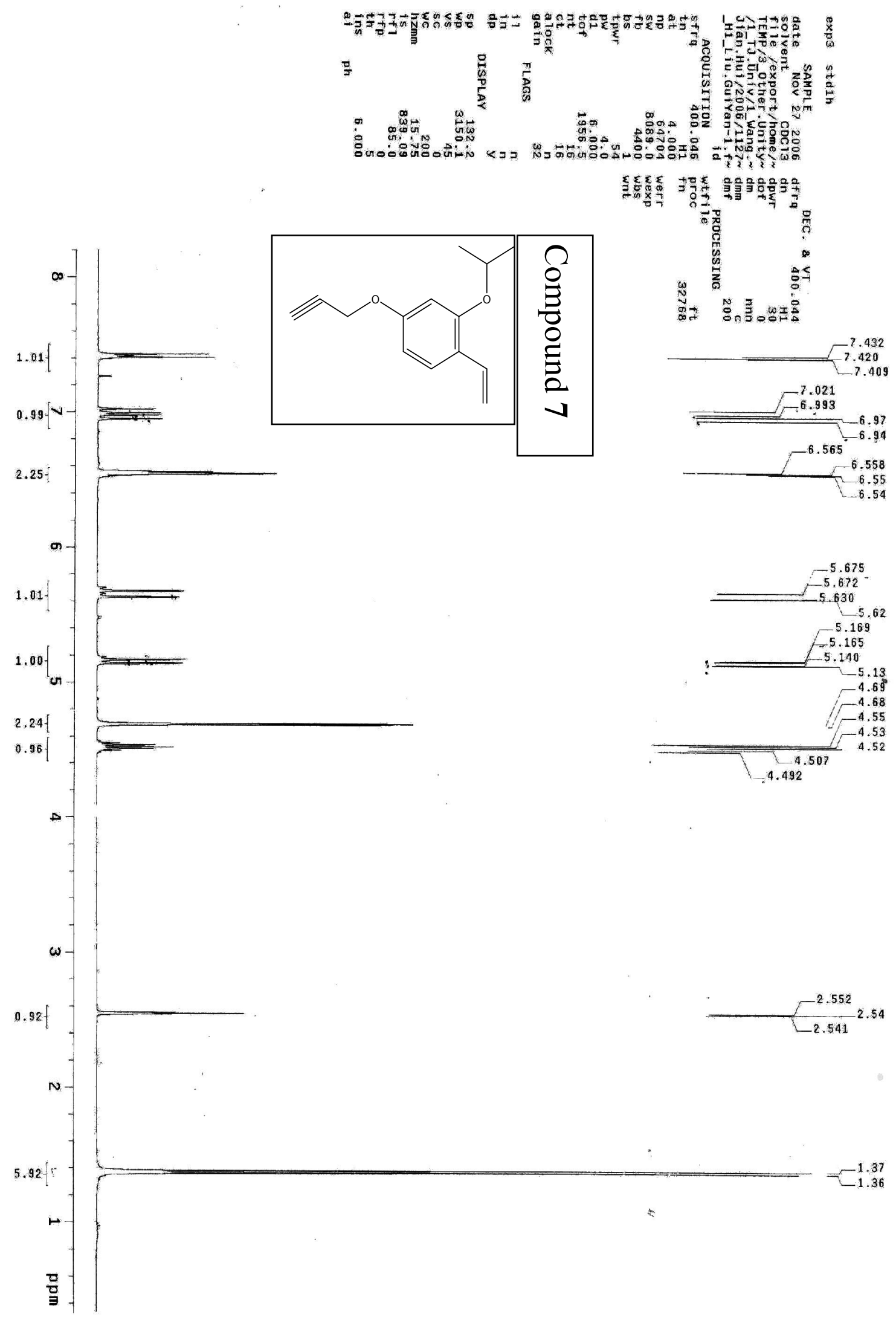




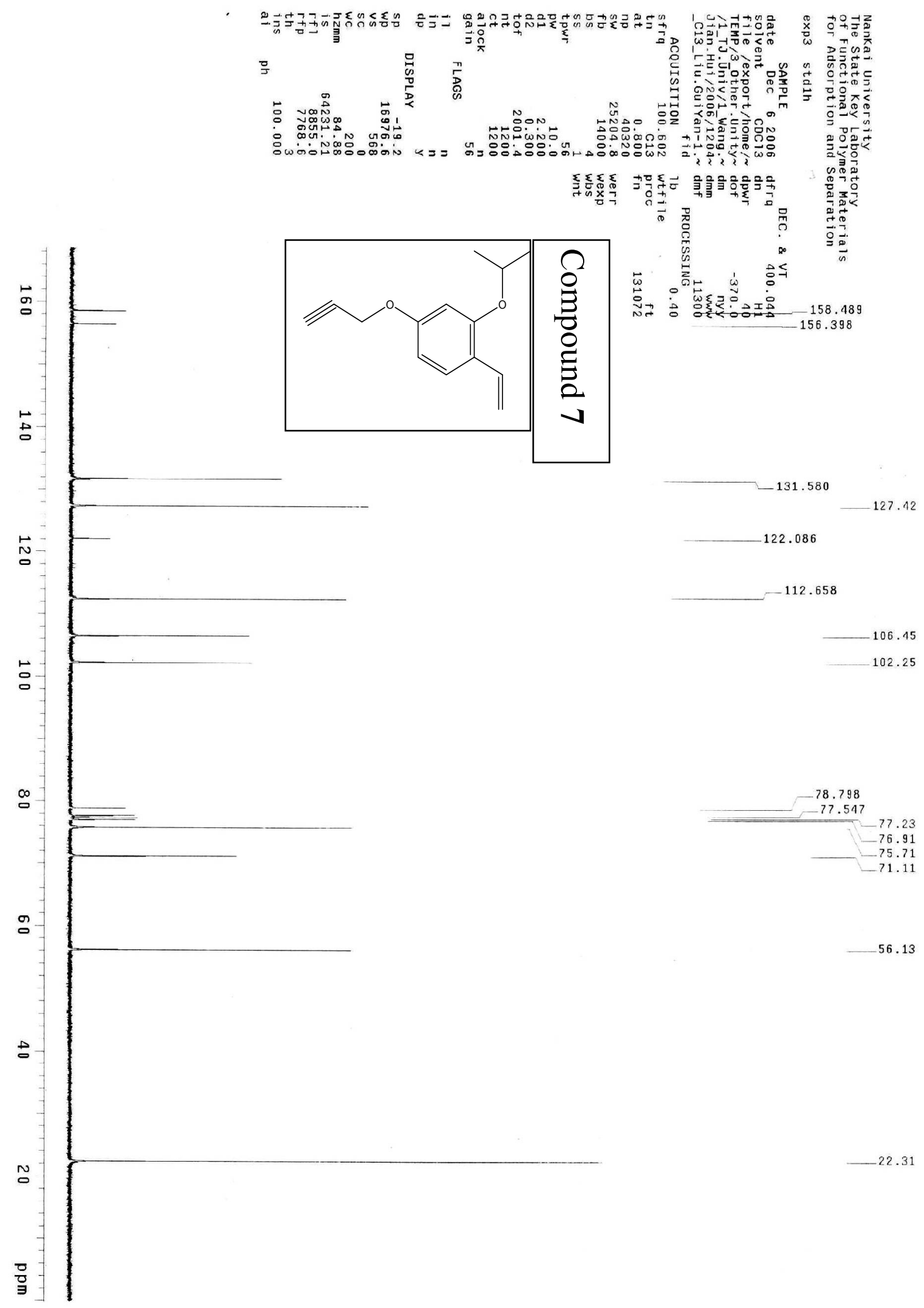









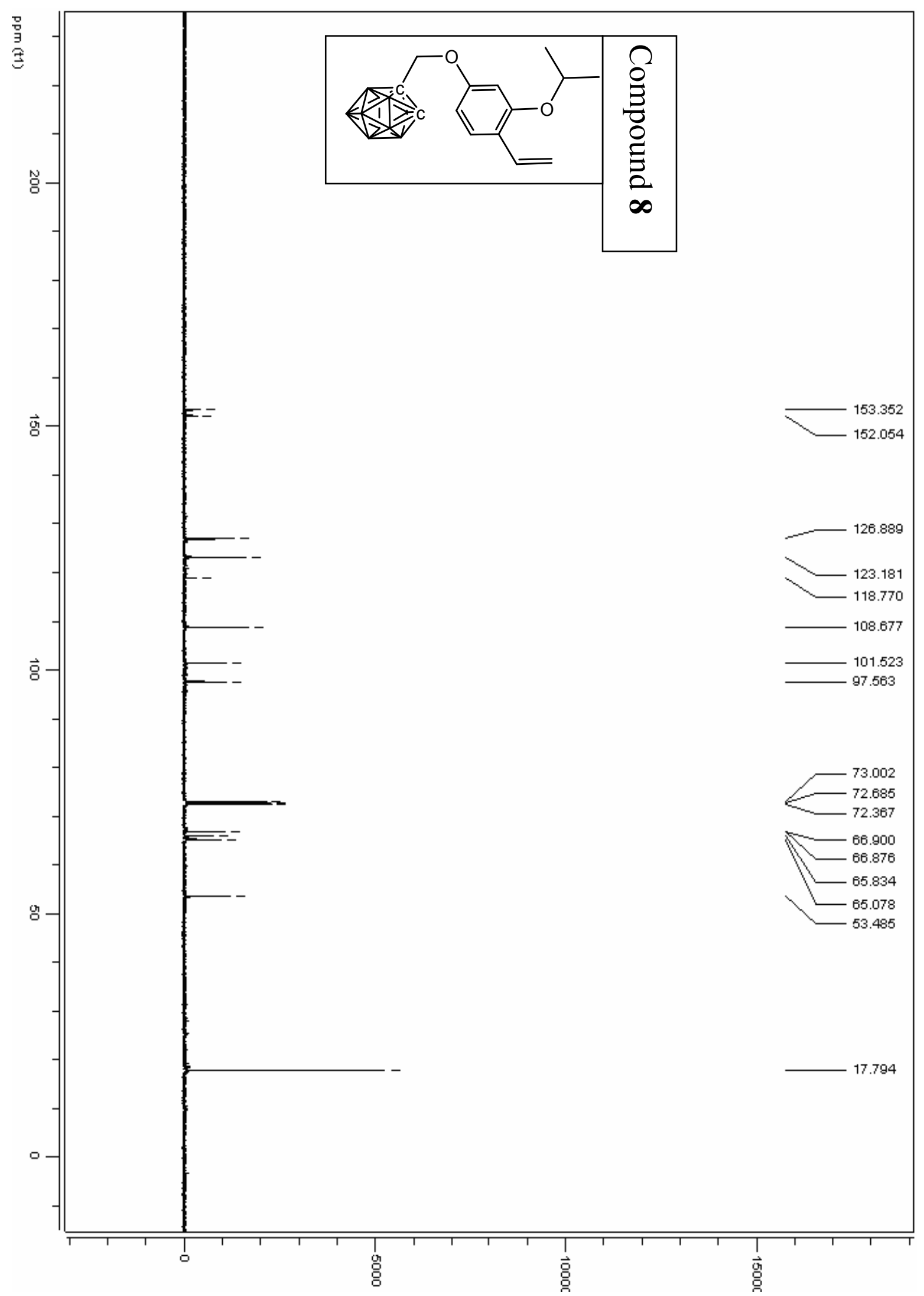



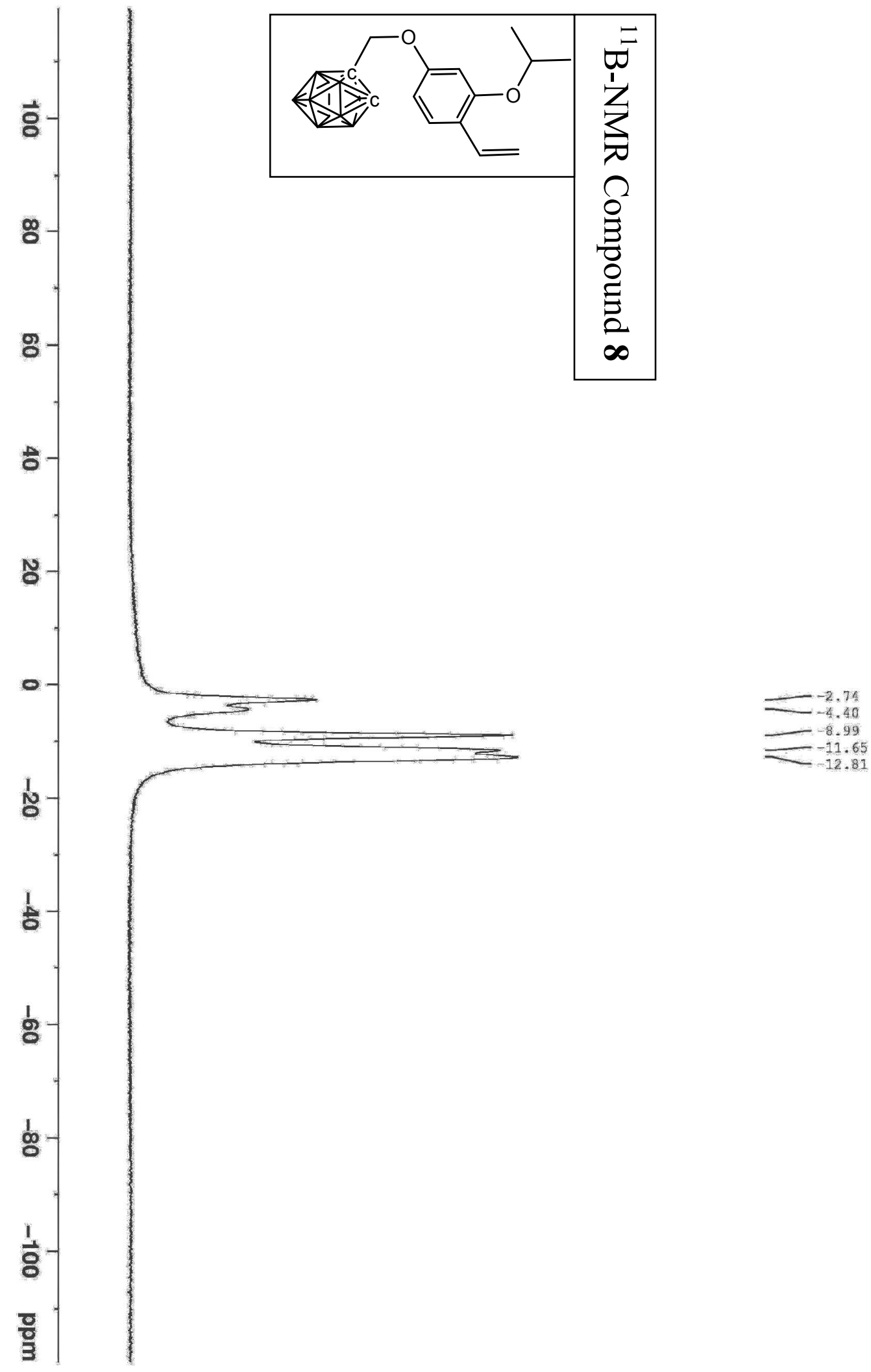


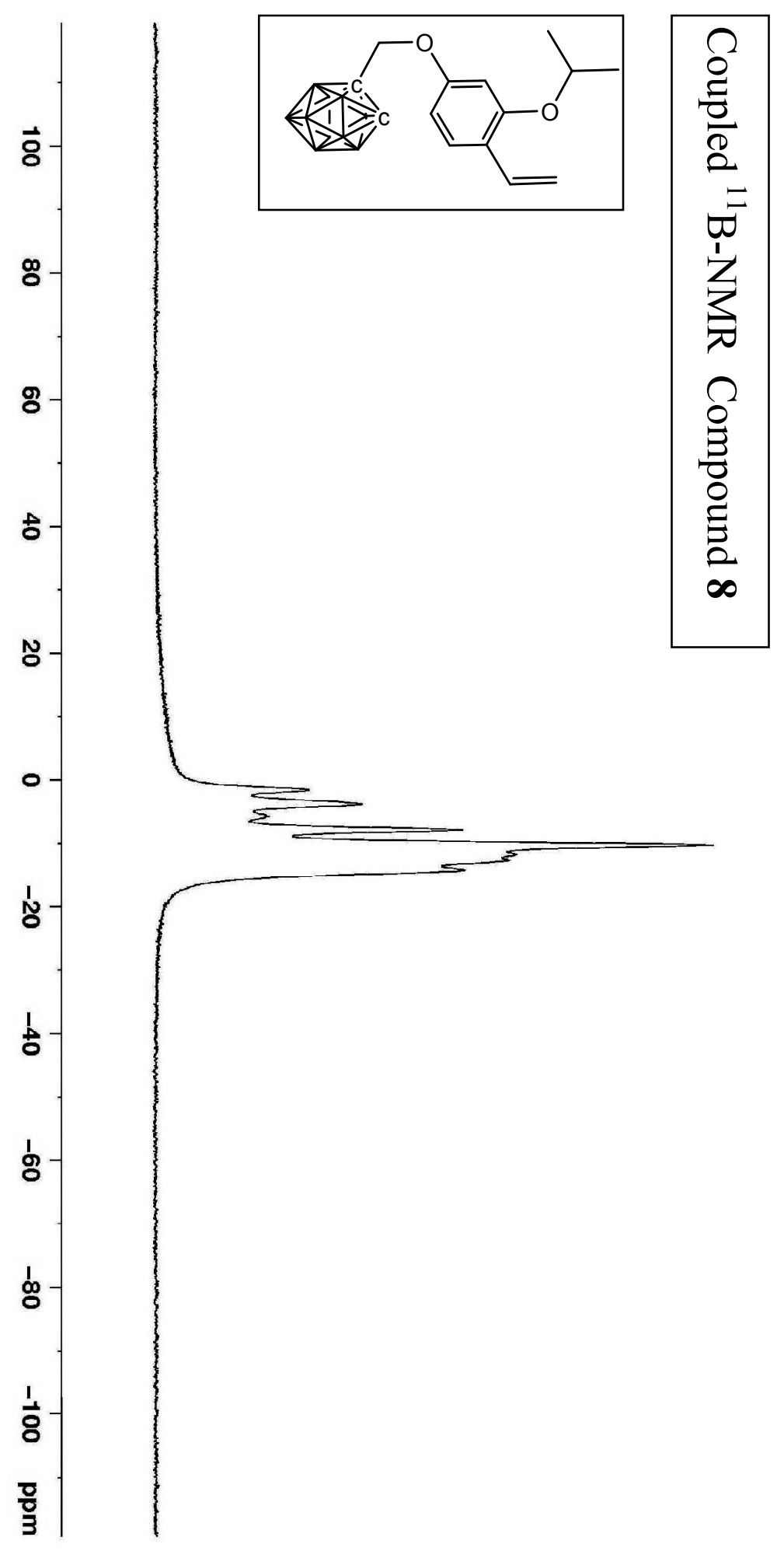




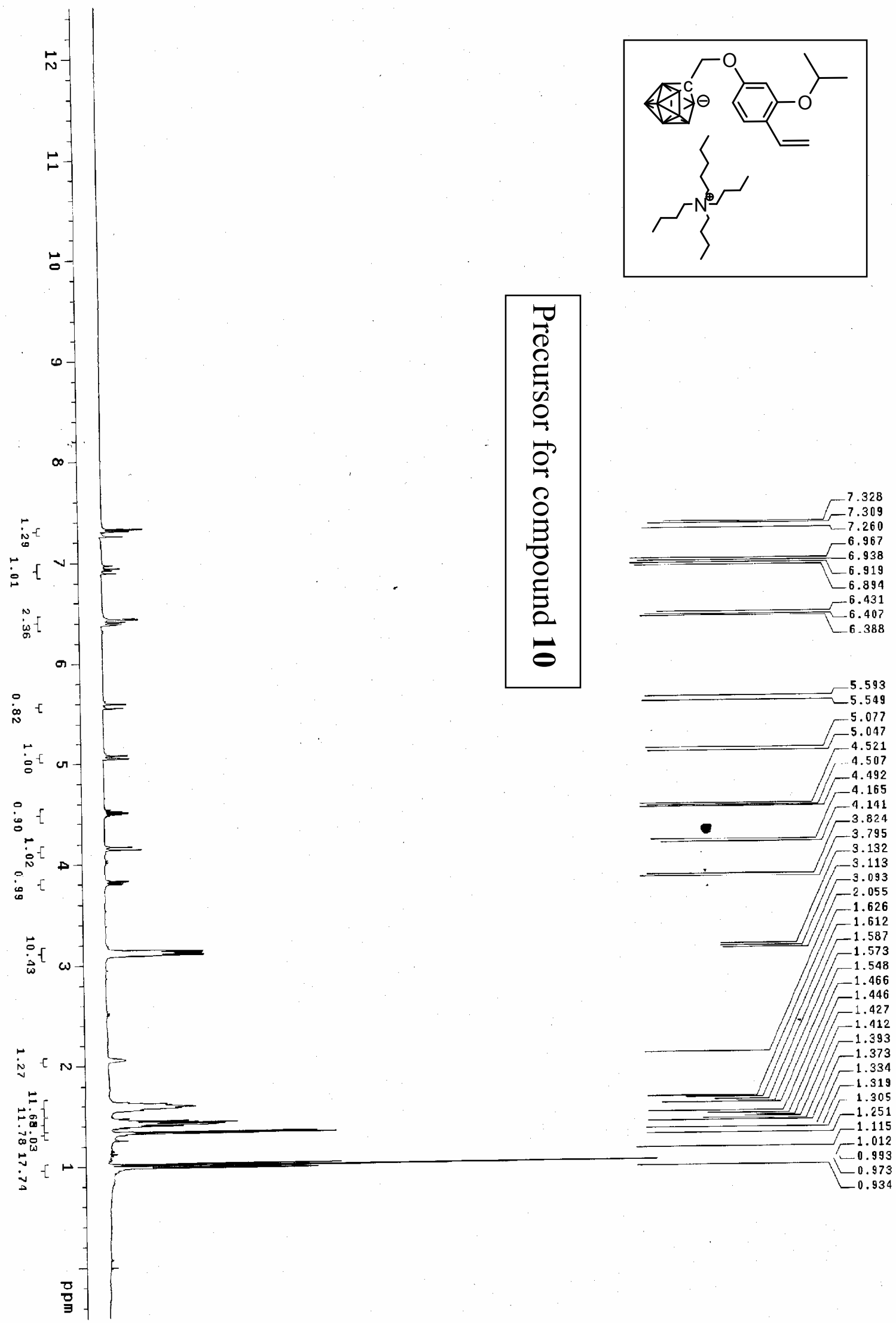




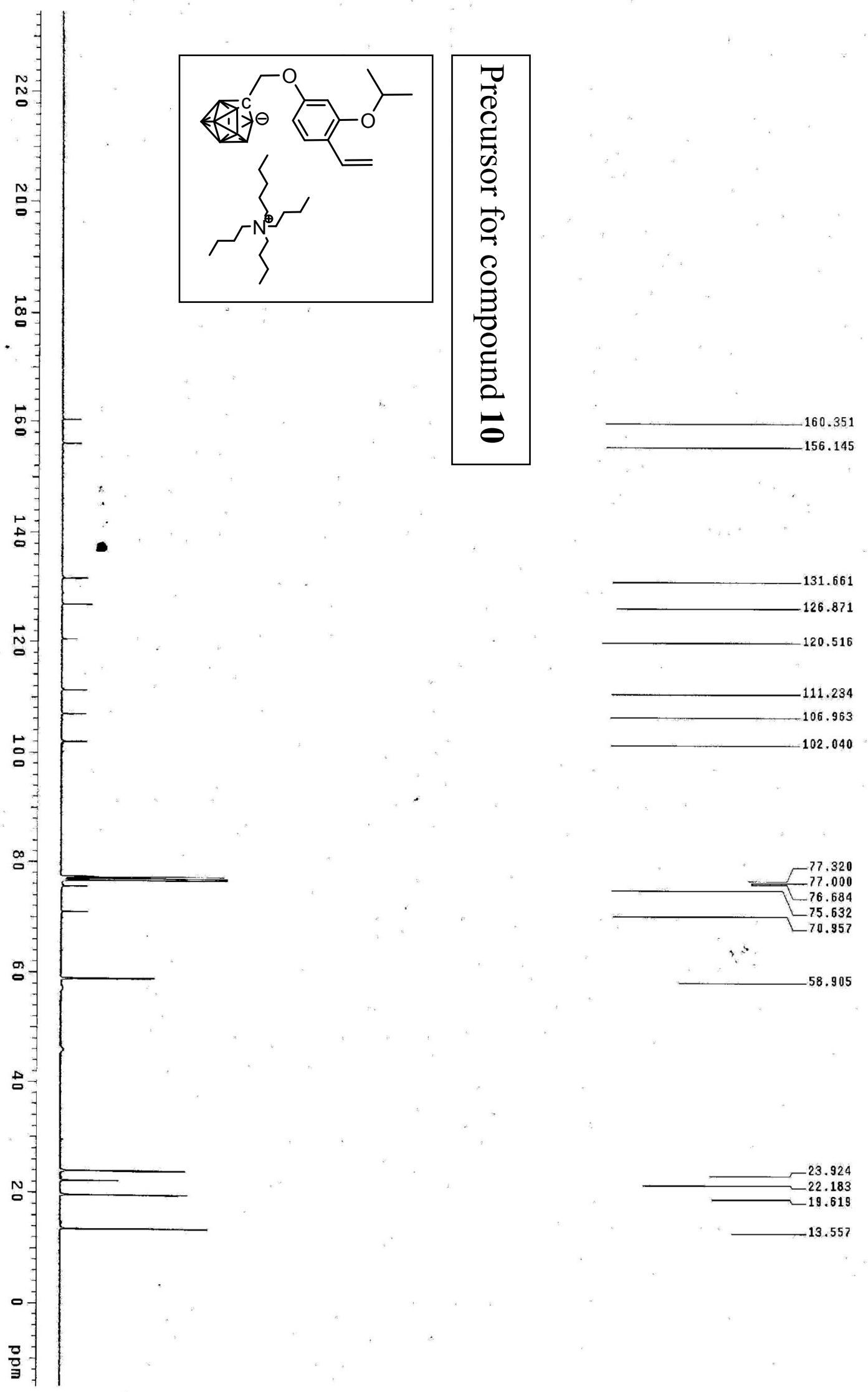




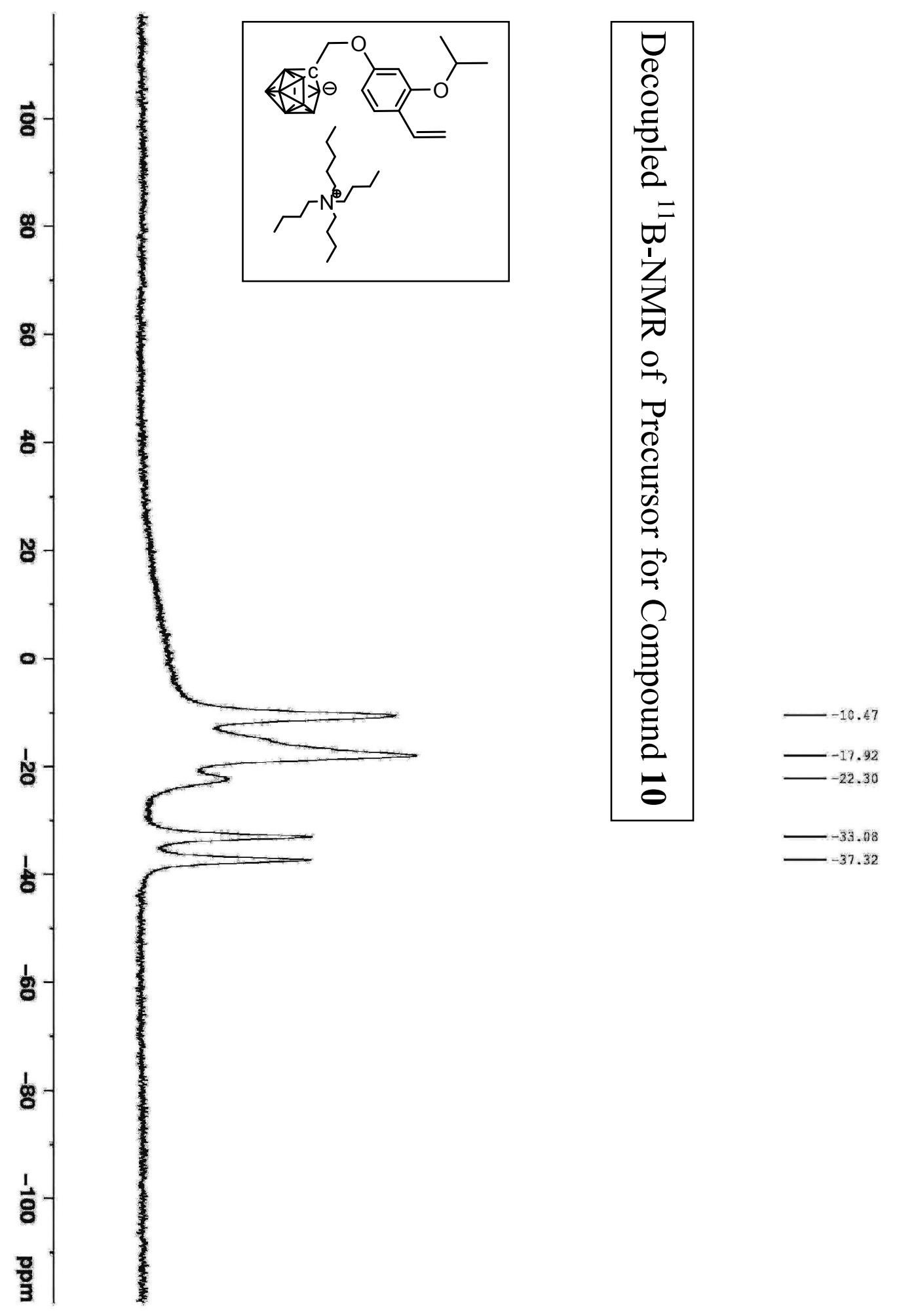




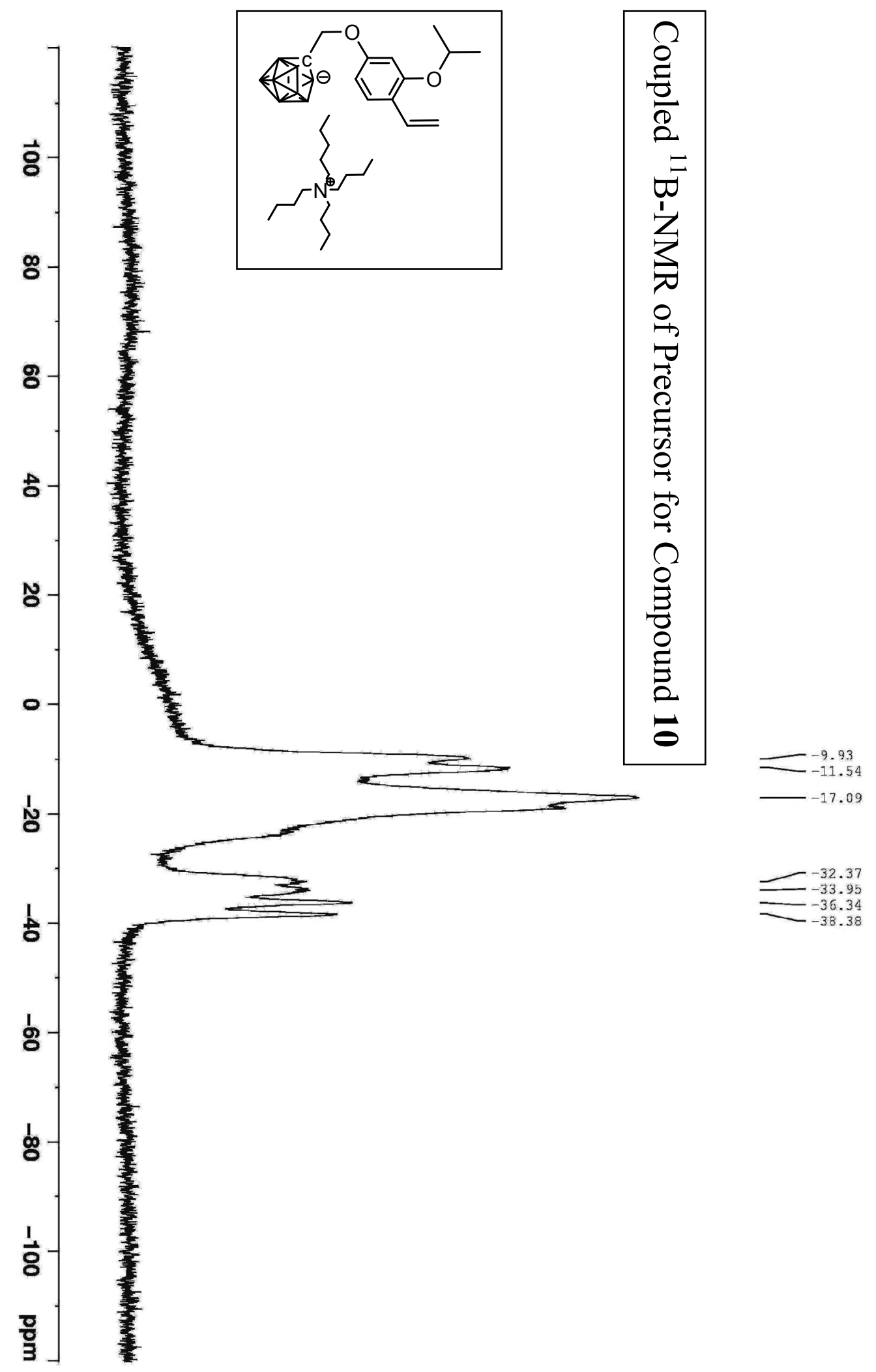




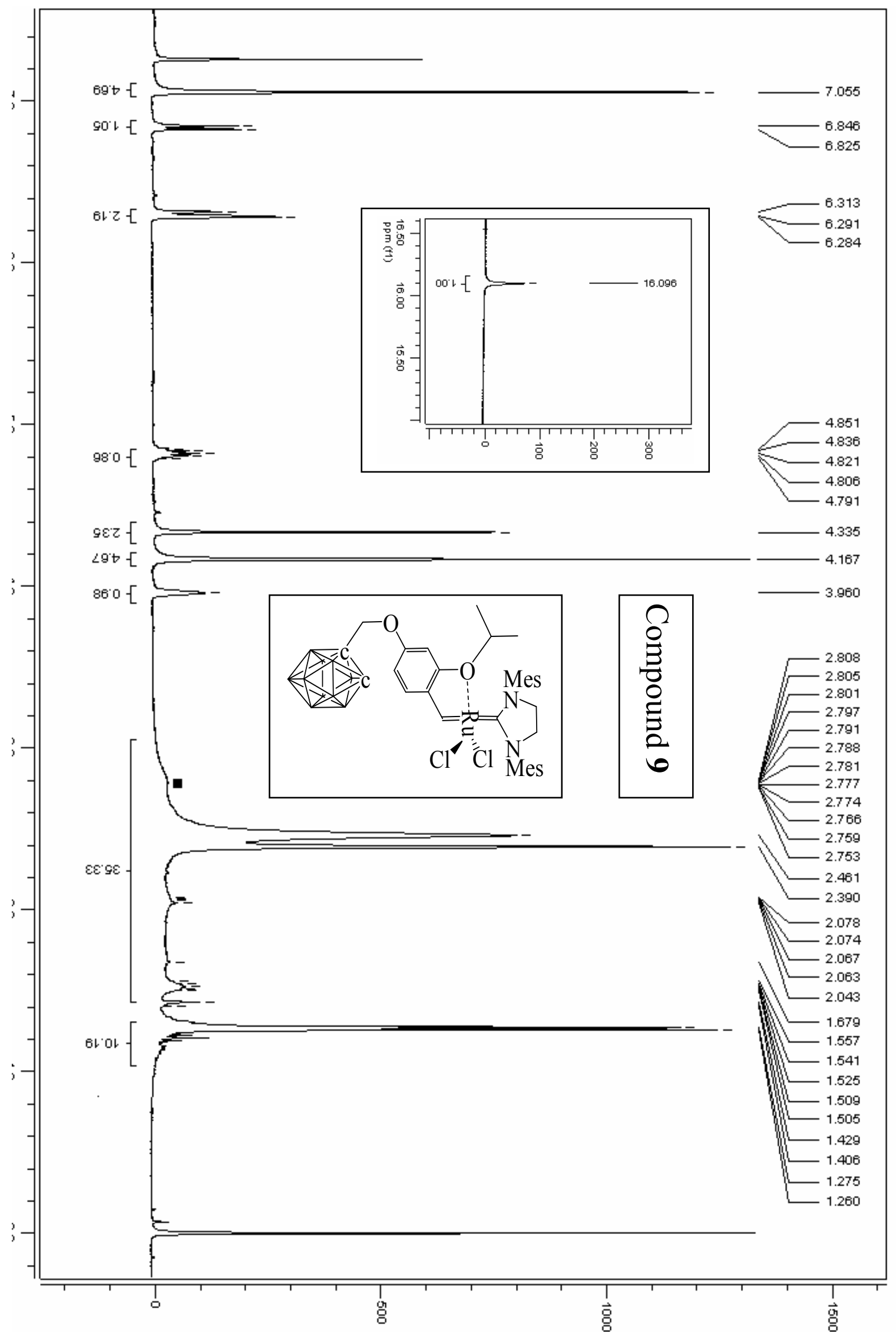




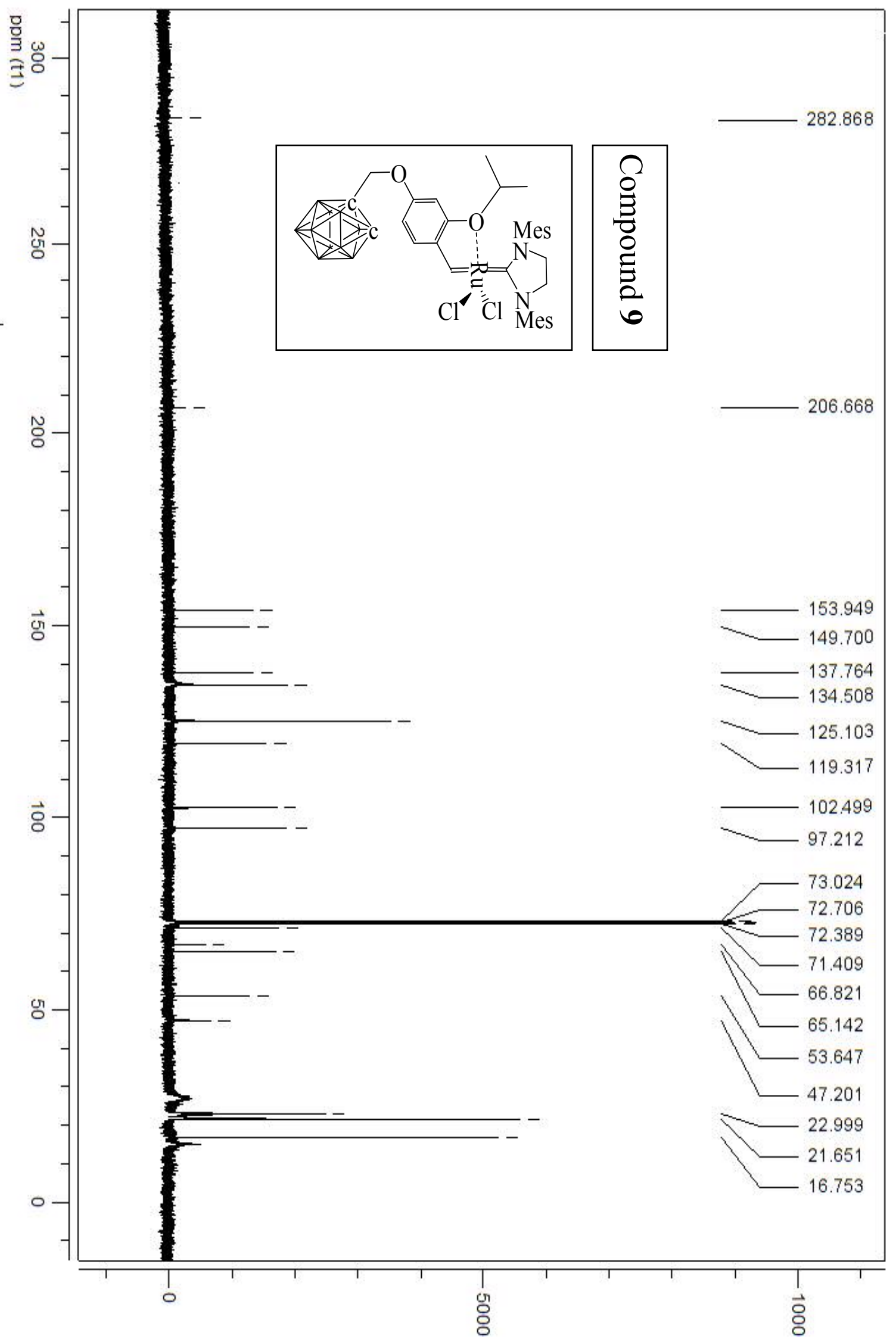




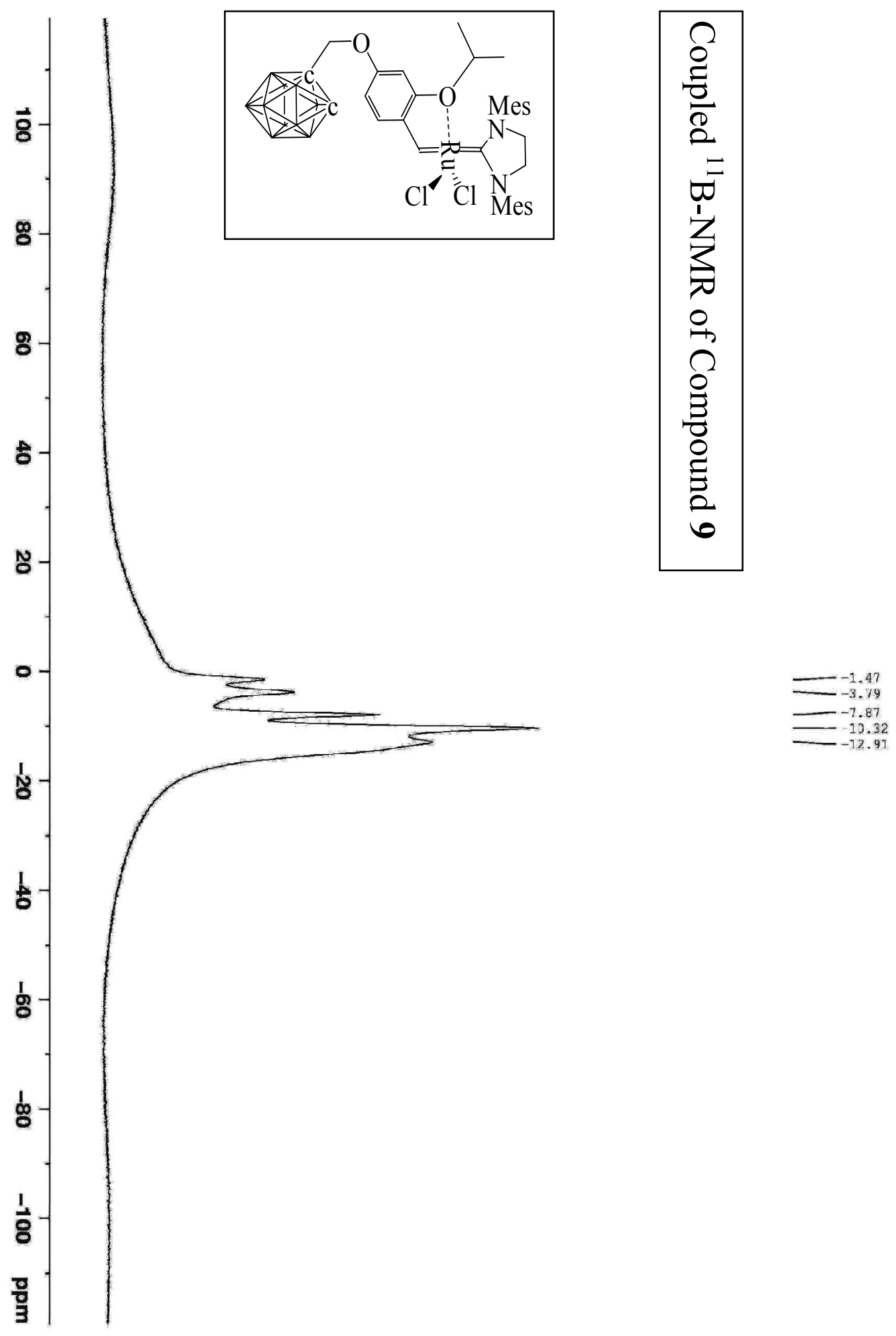




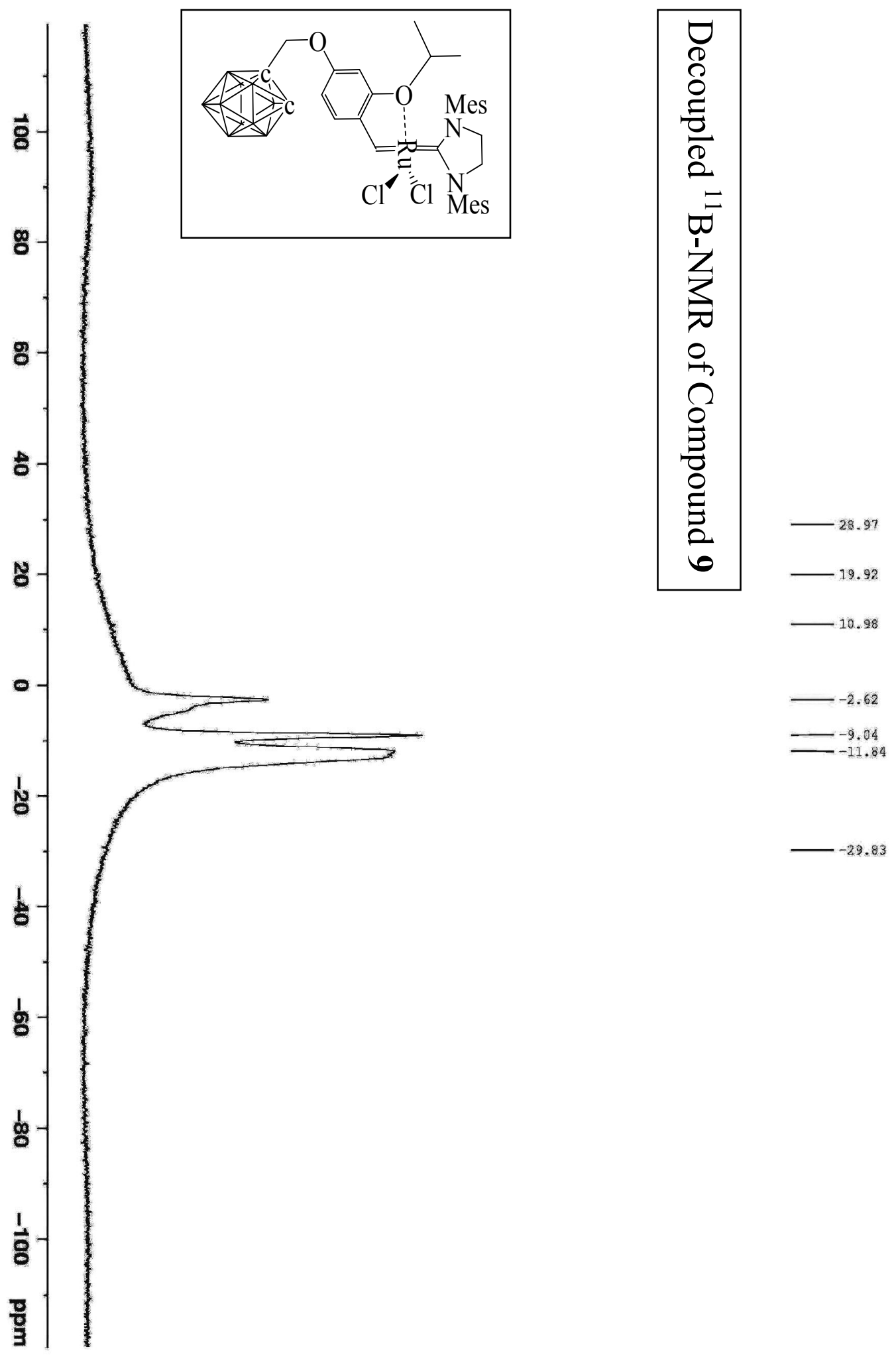




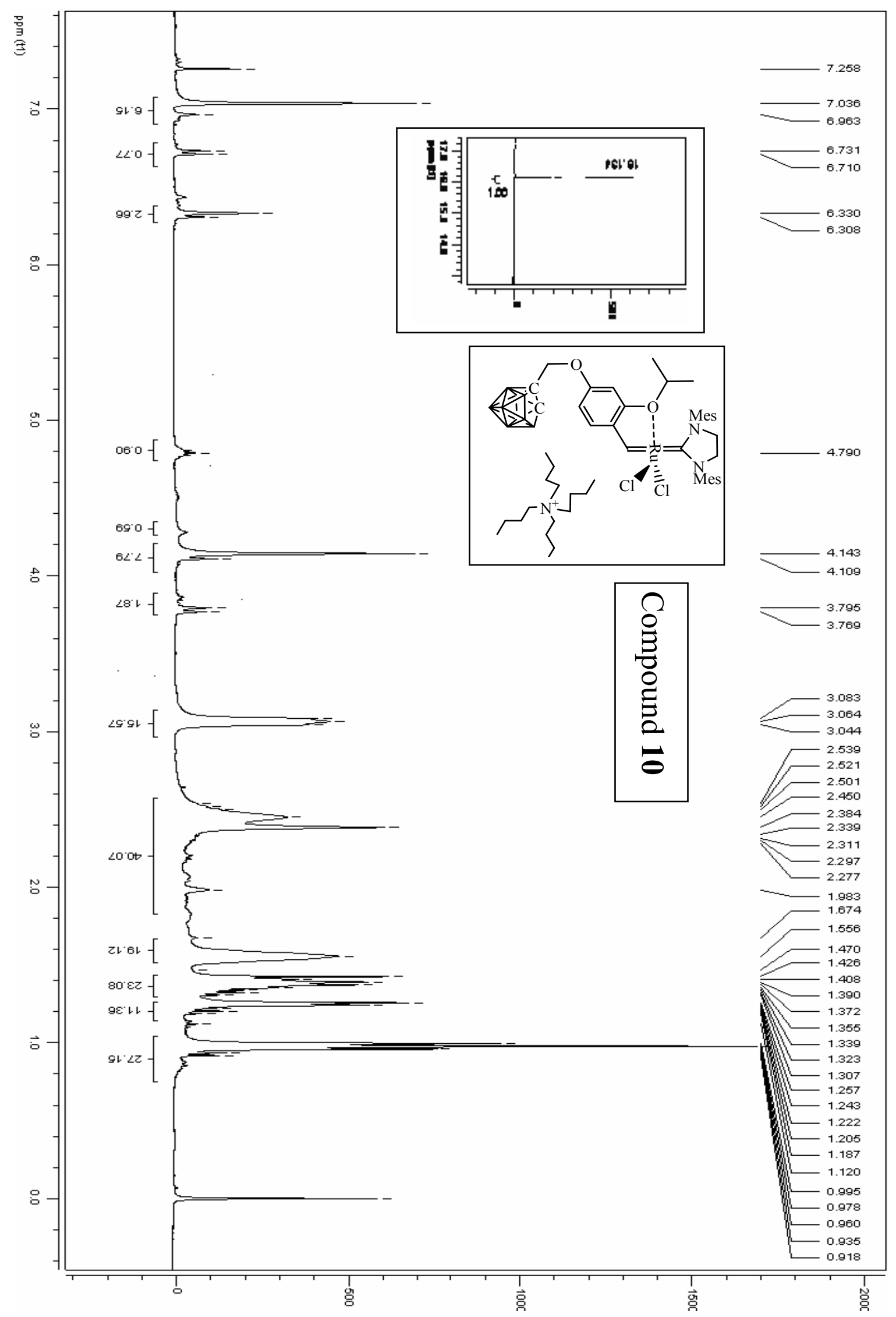




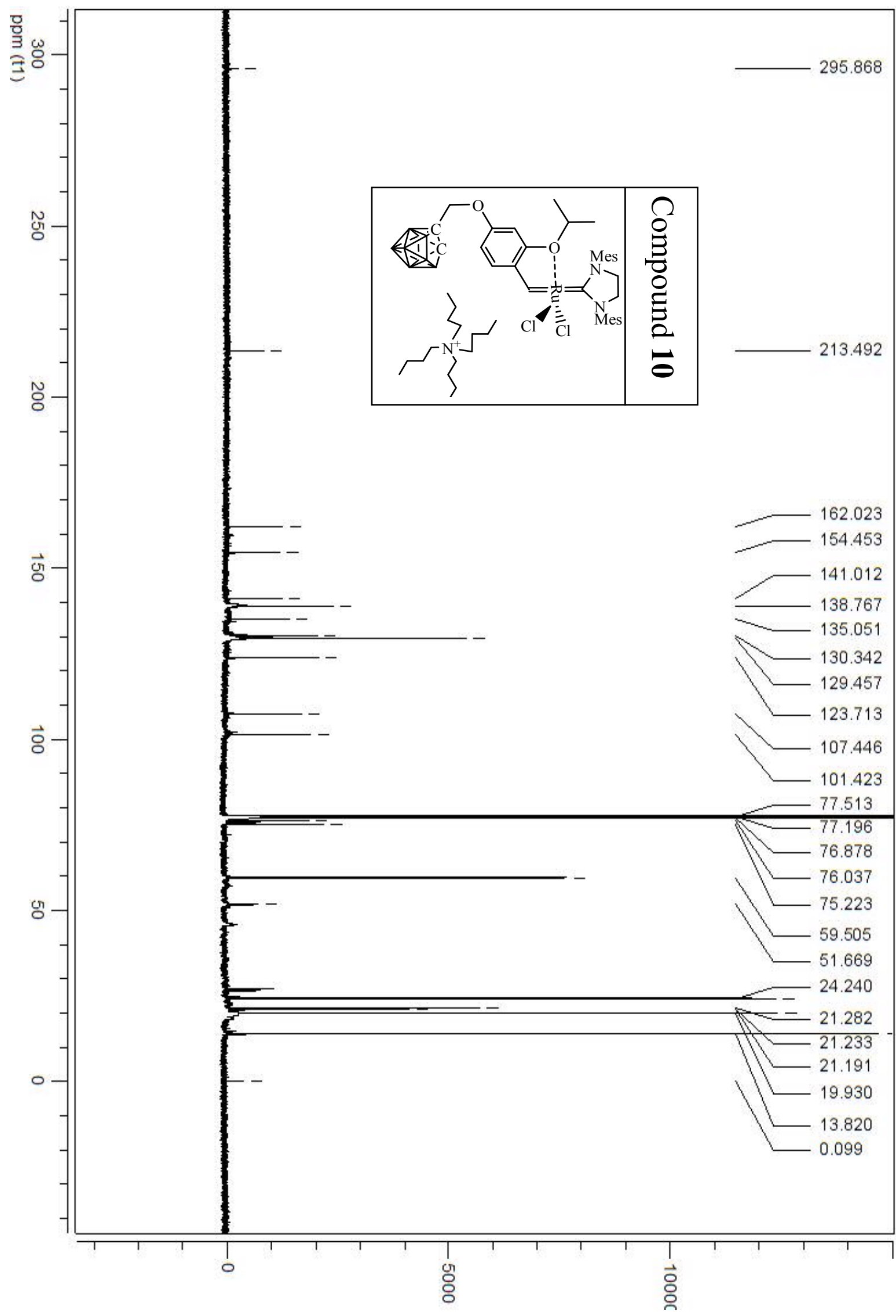




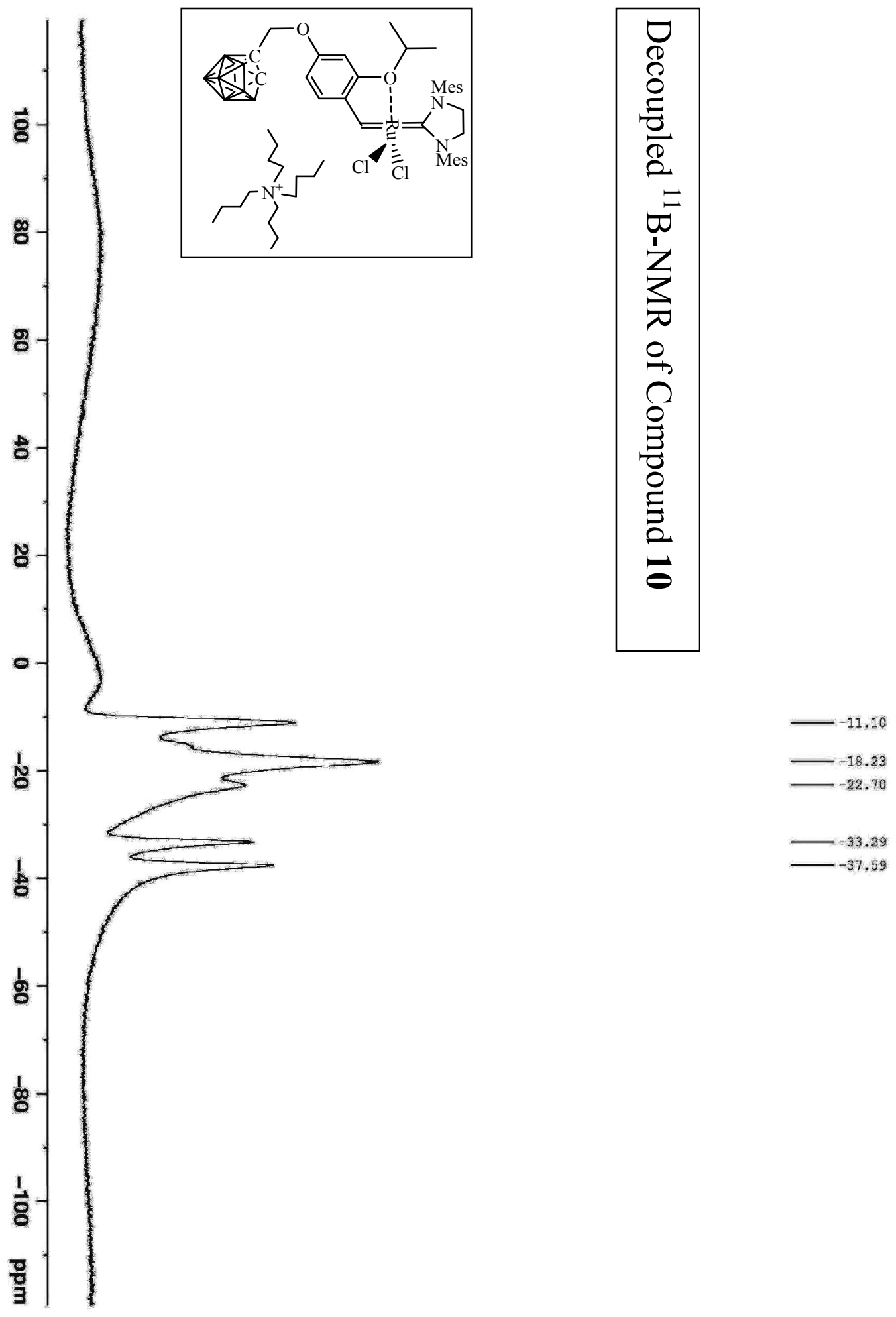




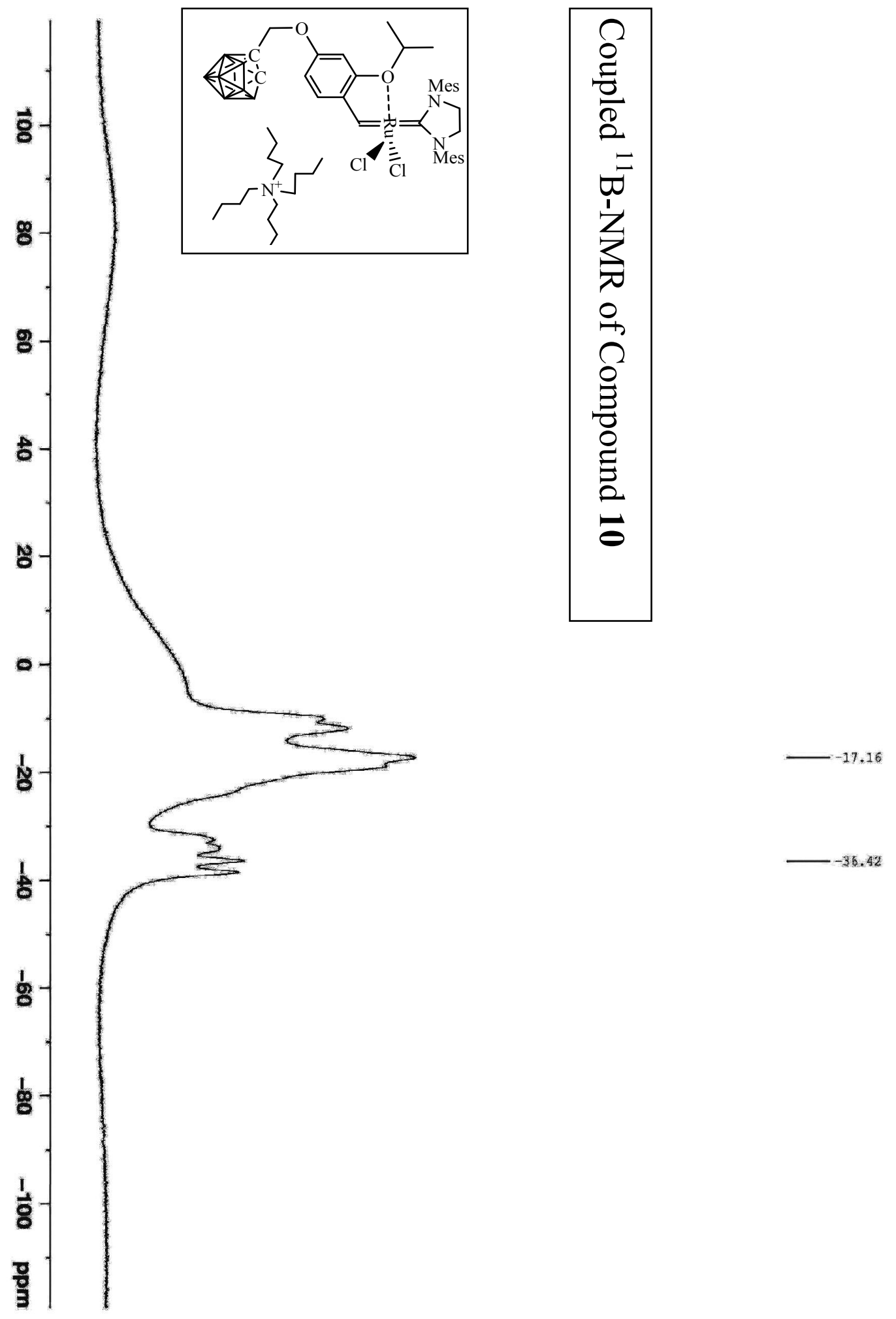

